\title{
Bootstrapping the three-loop hexagon
}

\author{
Lance J. Dixon, ${ }^{a, b}$ James M. Drummond ${ }^{a, c}$ and Johannes M. Henn ${ }^{d, e}$ \\ a PH-TH Division, CERN, \\ CH - 1211 Geneva 23, Switzerland \\ ${ }^{b}$ SLAC National Accelerator Laboratory, \\ 2575 Sand Hill Road, Menlo Park, CA 94025, U.S.A. \\ ${ }^{c}$ LAPTH, Université de Savoie, CNRS, \\ B.P. 110, F-74941 Annecy-le-Vieux Cedex, France \\ ${ }^{d}$ Institut für Physik, Humboldt-Universität zu Berlin, \\ Newtonstraße 15, D-12489 Berlin, Germany \\ ${ }^{e}$ Kavli Institute for Theoretical Physics, University of California, \\ Santa Barbara, CA 93106, U.S.A. \\ E-mail: lance@slac.stanford.edu, drummond@lapp.in2p3.fr, \\ jmhenn@ias. edu
}

ABStRaCt: We consider the hexagonal Wilson loop dual to the six-point MHV amplitude in planar $\mathcal{N}=4$ super Yang-Mills theory. We apply constraints from the operator product expansion in the near-collinear limit to the symbol of the remainder function at three loops. Using these constraints, and assuming a natural ansatz for the symbol's entries, we determine the symbol up to just two undetermined constants. In the multi-Regge limit, both constants drop out from the symbol, enabling us to make a non-trivial confirmation of the BFKL prediction for the leading-log approximation. This result provides a strong consistency check of both our ansatz for the symbol and the duality between Wilson loops and MHV amplitudes. Furthermore, we predict the form of the full three-loop remainder function in the multi-Regge limit, beyond the leading-log approximation, up to a few constants representing terms not detected by the symbol. Our results confirm an all-loop prediction for the real part of the remainder function in multi-Regge $3 \rightarrow 3$ scattering. In the multiRegge limit, our result for the remainder function can be expressed entirely in terms of classical polylogarithms. For generic six-point kinematics other functions are required.

KEYwords: Supersymmetric gauge theory, Extended Supersymmetry, Conformal and W Symmetry

ArXiv EPRINT: 1108.4461 


\section{Contents}

1 Introduction and outline 1

2 Pure functions and symbols $\quad 5$

$\begin{array}{lll}3 & \text { Constraining the three-loop remainder function } & 6\end{array}$

$\begin{array}{lll}4 & \text { OPE constraints } & 10\end{array}$

$\begin{array}{lll}5 & \text { Predictions for multi-Regge kinematics } & 15\end{array}$

6 Constraints on the final entry of the symbol 22

$\begin{array}{lll}7 & \text { Conclusions and outlook } & 25\end{array}$

A Change of variables between $u, v, w$ and $\tau, \sigma, \phi \quad 26$

B Change of variables between $u, v, w$ and $y_{u}, y_{v}, y_{w} \quad 27$

C Inversion-symmetric form of functions describing the multi-Regge limit 27

\section{Introduction and outline}

Scattering amplitudes in $\mathcal{N}=4$ super Yang-Mills theory (SYM) have fascinating properties, especially in the planar limit. One of their most surprising properties is an equivalence with certain light-like Wilson loop configurations, for which there is strong empirical evidence at weak coupling, as well as general arguments originating from strong coupling [1-8]. The equivalence relates the suitably-defined finite parts of maximally-helicity-violating (MHV) scattering amplitudes to the finite parts of Wilson loops evaluated on a null polygonal contour in dual (or region) space. The edges of the polygon are defined by the gluon momenta $p_{i}^{\mu}$ via

$$
p_{i}^{\mu}=x_{i}^{\mu}-x_{i+1}^{\mu} .
$$

The contour has corners (or cusps) at the points $x_{i}$. The equivalence between amplitudes and Wilson loops implies that the analytic properties of Wilson loops in the dual space can be identified with those of scattering amplitudes in momentum space.

Wilson loops in a conformal field theory exhibit conformal symmetry. The null polygonal Wilson loops related to scattering amplitudes are ultraviolet divergent due to the presence of cusps on the contour. Nonetheless they still obey a conformal Ward identity $[4,9]$. This identity can be simply stated as follows. We write the logarithm of the Wilson loop with $n$ cusps as a sum of divergent and finite terms,

$$
\log W_{n}=[\mathrm{UV} \text { divergent }]_{n}+F_{n}^{\mathrm{WL}} .
$$


The Ward identity for the finite part is then

$$
K^{\mu} F_{n}^{\mathrm{WL}}=-\frac{\gamma_{K}}{2} \sum_{i=1}^{n}\left(2 x_{i}^{\mu}-x_{i-1}^{\mu}-x_{i+1}^{\mu}\right) \log x_{i-1, i+1}^{2},
$$

where $x_{i, j}=x_{i}-x_{j}$ and $x_{i+n} \equiv x_{i}$, and $K^{\mu}$ are the generators of (dual) special conformal transformations,

$$
K^{\mu}=\sum_{i=1}^{n}\left[2 x_{i}^{\mu} x_{i}^{\nu} \frac{\partial}{\partial x_{i}^{\nu}}-x_{i}^{2} \frac{\partial}{\partial x_{i \mu}}\right] .
$$

The cusp anomalous dimension [10] $\gamma_{K}$ is predicted to all orders in the coupling constant [11].

The Ward identity (1.3) fixes $F_{n}^{\mathrm{WL}}$, up to functions of conformally invariant cross ratios. Below six points there are no such cross-ratios and the solution is unique up to an additive constant. In fact this solution coincides precisely with the BDS ansatz [12] for the finite part of MHV scattering amplitudes. At six points and beyond there are cross ratios, so the solution is not unique. The BDS ansatz still provides a particular solution to the Ward identity for all $n$, but it does not give the complete answer. A convenient way of writing the solution to the Ward identity is then

$$
F_{n}^{\mathrm{WL}}=\gamma_{K} F_{n, 1-\text { loop }}^{\mathrm{WL}}+R_{n}
$$

Here $F_{n, 1-\text { loop }}^{\mathrm{WL}}$ is the one-loop result for $F_{n}^{\mathrm{WL}}$, while $R_{n}$ is the 'remainder function', which is a function only of conformal cross ratios and becomes non-vanishing at two loops [5]. In terms of the loop expansion parameter $a \equiv g^{2} N_{c} /\left(8 \pi^{2}\right)$, the remainder function is expanded as

$$
R_{n}=\sum_{l=2}^{\infty} a^{l} R_{n}^{(l)}
$$

At six points, the remainder function depends on three dual conformal cross ratios,

$$
u=\frac{x_{13}^{2} x_{46}^{2}}{x_{14}^{2} x_{36}^{2}}=\frac{s_{12} s_{45}}{s_{123} s_{345}}, \quad v=\frac{x_{24}^{2} x_{15}^{2}}{x_{25}^{2} x_{14}^{2}}=\frac{s_{23} s_{56}}{s_{234} s_{456}}, \quad w=\frac{x_{35}^{2} x_{26}^{2}}{x_{36}^{2} x_{25}^{2}}=\frac{s_{34} s_{61}}{s_{345} s_{561}},
$$

which are in turn built from the Lorentz invariants $s_{i, j}=\left(p_{i}+p_{j}\right)^{2}$ and $s_{i, j, k}=\left(p_{i}+p_{j}+p_{k}\right)^{2}$. The gluon momenta for the scattering process, $p_{i}^{\mu}$ with $i=1,2, \ldots, 6$, satisfy the on-shell conditions $p_{i}^{2}=0$.

The conformal symmetry of the Wilson loop implies that the dual planar MHV amplitudes exhibit 'dual conformal symmetry'. This symmetry has been observed in the form of the scattering amplitudes in many guises: the form of the integrals in the perturbative expansion [13-15]; the background isometry of the AdS sigma model after Tduality $[1,8,16]$; the structure of tree-level amplitudes, where it extends to dual superconformal symmetry [17], and combines with the original Lagrangian superconformal symmetry to form a Yangian symmetry [18]; the structure of the scattering amplitudes on the Coulomb branch [19] and in higher dimensions [19-22]; and the form of the on-shell recursion relations for the four-dimensional planar integrand [23]. Many review articles are 
available on different aspects of all of these developments, including refs. [24-31]. For the purposes of this paper the important point is simply that the Ward identity (1.3) requires the function $R_{6}$ to depend only on the invariant cross ratios $u, v$ and $w$.

Much recent progress [32-36] has focused on understanding the structure of the remainder function, in part due to the fact that this same function governs the structure of scattering amplitudes, both at strong coupling [1] and in the MHV sector in perturbation theory [5-7]. Understanding its form then promises to greatly enhance our understanding of scattering amplitudes in general. A very important result in this direction was the analytic calculation of the Feynman integrals appearing at two loops in the hexagonal Wilson loop [32, 33], which provided a closed-form expression for the remainder function in terms of (many) multi-dimensional polylogarithms, or Goncharov polylogarithms. Remarkably, this seemingly complicated expression could be dramatically simplified into a few lines of classical polylogarithms [35]. An important tool for finding such a compact form of the two-loop, six-point remainder function is the notion of the symbol of a transcendental function [37]. The symbol is a quantity which preserves the underlying algebraic nature of the function, while forgetting about certain analytic properties, such as the particular branch cut on which the function should be evaluated. Complicated identities between polylogarithms reduce to simple algebraic relations at the level of symbols. The symbol can therefore be a key step in discerning the analytic structure of amplitudes. For example, a conjecture has been made recently for the symbol of the two-loop remainder function for an arbitrary number of points [38]. Of course, eventually one would like to reconstruct the actual function represented by the symbol.

Another important property of polygonal Wilson loops is that they should respect a particular operator product expansion (OPE) in the region where several consecutive edges are nearly collinear $[39,40]$. This idea has recently been used to argue that at two loops the hexagon remainder function can be uniquely fixed from the knowledge only of the leading corrections to the energies of the exchanged states in the OPE [41]. The OPE has also recently been used to address the same problem for Wilson loops with more than six sides [42], and for super Wilson loops associated with non-MHV amplitudes [43].

An important kinematical limit of higher-point scattering amplitudes is the multiRegge limit. This limit is a generalization of the high-energy limit of four-point scattering, but is one in which multiple parameters can survive, related to the ordering of the final-state particles in rapidity. For the MHV amplitudes in planar $\mathcal{N}=4$ super Yang-Mills theory, this structure has been explored in several papers [44-50]. Indeed, this limit provided early evidence that the BDS ansatz needed to be corrected at two loops, starting with the sixpoint amplitude [44]. While the remainder function $R_{6}(u, v, w)$ vanishes in the Euclidean version of multi-Regge kinematics [51-53], in the physical region its discontinuity is nonzero and can be analysed. When dual conformal invariance holds, this discontinuity depends nontrivially on two dimensionless variables, rather than the three variables $u, v$ and $w$ characterizing generic kinematics.

A consequence of the duality between MHV amplitudes and Wilson loops is that the multi-Regge behaviour of the amplitude should be consistent with the OPE behaviour of the Wilson loop in the near-collinear limit. That is, there is a further limit one can 
take of the multi-Regge kinematics which is collinear in nature. This combined limit was studied recently [50], and it was shown that constraints from the two limits pass a selfconsistency test.

In this paper, inspired by all these exciting recent developments, we will make an ansatz for the symbol of the three-loop hexagon remainder function, $R_{6}^{(3)}(u, v, w)$, which is heavily constrained by the structures described above. We are able to apply all of the physical requirements, such as the correct collinear behaviour, OPE expansion, multiRegge limits and so on, at the level of the symbol. The correct near-collinear behaviour, governed by the OPE expansion, is one of the strongest constraints on our ansatz. It is quite non-trivial that there is a consistent solution to the combined constraints. For general kinematics, the solution for the symbol is not unique, but contains 26 arbitrary constants. However, all but three of these parameters are irrelevant in the multi-Regge limit. Analysing the symbol in this limit, and imposing consistency with the leading logarithmic prediction [48], we find that two of the three parameters relevant in this limit can be fixed. Hence the symbol is completely fixed, up to a single constant parameter, in this regime. An additional constraint enables us to show that this extra constant parameter actually vanishes. The latter constraint is an all-loop-order prediction [49] concerning the behaviour of the real part of the remainder function in the multi-Regge limit, after analytic continuation to $3 \rightarrow 3$ kinematics. We have found functions corresponding to the symbol in this limit, and we constrain the possible beyond-the-symbol ambiguities in term of a few additional constants. These functions are all expressible in terms of classical logarithms and polylogarithms. Thus we are able to make new, rather non-trivial predictions for the next-to-leading and next-to-next-to-leading logarithmic approximations to the scattering of six gluons at three loops in the multi-Regge limit.

We then examine the implications of imposing a further requirement on the form of the final entries of our symbol. This restriction constrains the derivatives of the remainder function. It can be motivated by the differential equations obeyed by one-loop [54-56] and multi-loop integrals [57] related to scattering amplitudes in planar $\mathcal{N}=4$ super-Yang-Mills theory. The same restriction has also been identified within a supersymmetric formulation of the Wilson loop [38]. We find that imposing this final-entry condition fixes the symbol completely up to just two free parameters, and furthermore it determines the symbol uniquely in the multi-Regge limit, and consistently with the all-loop-order prediction for $3 \rightarrow 3$ scattering.

The paper is organised as follows. Section 2 contains a brief review of pure functions and properties of their associated symbols. In section 3 we make an ansatz for the symbol of the remainder function of a particular, natural form, and we describe the constraints that it must satisfy in order to be consistent. In section 4 we discuss the most involved constraints, namely the ones coming from certain leading terms in the OPE expansion. Our focus is on the interesting case of the hexagon at three loops. We find that our ansatz is consistent with all of the constraints we apply, and indeed there is a 26-parameter solution at this stage. In section 5 we analyse our symbol in multi-Regge kinematics, and produce new expressions for the next-to-leading and next-to-next-to-leading logarithmic approximations at three loops. In section 6 we discuss the condition we impose on the final 
entry of the symbol, and describe how it reduces our ansatz to just two free parameters. We also remark that for generic values of $u, v$ and $w$, the three-loop remainder function cannot be described in terms of classical polylogarithms, in contrast to what happened at two loops. In section 7 we present our conclusions and give a brief outlook. Three appendices give some useful relations between different sets of kinematic variables, as well as an alternate representation of the logarithmic coefficients in the multi-Regge limit.

In additional files accompanying this article, as both Mathematica notebooks and plain text files, we provide the symbol for the three-loop remainder function, after imposing the final-entry constraint. We also provide the symbols associated with the remainder function in the multi-Regge limit.

\section{Pure functions and symbols}

The remainder function of $\mathcal{N}=4 \mathrm{SYM}$ is expected to be described in terms of pure functions. We define a pure function of degree (or weight) $k$ recursively, by demanding that its differential satisfies

$$
d f^{(k)}=\sum_{r} f_{r}^{(k-1)} d \log \phi_{r}
$$

Here the sum over $r$ is finite and $\phi_{r}$ are algebraic functions. This recursive definition is for all positive $k$; the only degree zero pure functions are constants. The definition (2.1) includes logarithms and classical polylogarithms, as well as other iterated integrals, such as harmonic polylogarithms of one [58] or more [59-62] variables.

The symbol [37] $\mathcal{S}(f)$ of a pure function $f$ is defined recursively with respect to eq. (2.1),

$$
\mathcal{S}\left(f^{(k)}\right)=\sum_{r} \mathcal{S}\left(f_{r}^{(k-1)}\right) \otimes \phi_{r}
$$

If we continue this process until we reach degree 0 , we find that $\mathcal{S}\left(f^{(k)}\right)$ is an element of the $k$-fold tensor product of the space of algebraic functions,

$$
\mathcal{S}\left(f^{(k)}\right)=\sum_{\vec{\alpha}} \phi_{\alpha_{1}} \otimes \ldots \otimes \phi_{\alpha_{k}}
$$

where $\vec{\alpha} \equiv\left\{\alpha_{1}, \ldots, \alpha_{k}\right\}$. The symbol of a function does not contain all the information about the function. In particular, it loses information about which logarithmic branch the integrand of an iterated integral is on, at each stage of integration. It also does not detect functions that are transcendental constants multiplied by pure functions of lower degree. (That is, such functions have zero symbol.) The symbol therefore corresponds to an equivalence class of functions that differ in these aspects. Nevertheless, the symbol is extremely useful, because complicated identities between transcendental functions defined by iterated integrals become simple algebraic identities.

If a symbol can be expressed as a sum of terms, with all entries in each term belonging to a given set of variables, then we say that the symbol can be factorised in terms of that 
set of variables. From the definition of the symbol, a term containing an entry which is a product can be split into the sum of two terms, according to

$$
\ldots \otimes \phi_{1} \phi_{2} \otimes \ldots=\ldots \otimes \phi_{1} \otimes \ldots+\ldots \otimes \phi_{2} \otimes \ldots
$$

Performing this factorisation is usually necessary to identify all algebraic relations between terms. It is often necessary to perform the step again after taking a kinematic limit, because the algebraic relations in the limit are different than for generic kinematics.

The elements of the symbol are not all independent. In particular the integrability condition $d^{2} f^{(k)}=0$ for any function implies relations among the different elements. These relations can be described simply: One picks two adjacent slots in the symbol $\phi_{\alpha_{i}} \otimes \phi_{\alpha_{i+1}}$ and replaces the corresponding elements by the wedge product $d \log \phi_{\alpha_{i}} \wedge d \log \phi_{\alpha_{i+1}}$ in every term. The resulting expression must vanish.

It is very helpful in our analysis to consider the discontinuities of the functions involved. The symbol makes clear the locations of the discontinuities of the function. If we have

$$
\mathcal{S}\left(f^{(k)}\right)=\sum_{\vec{\alpha}} \phi_{\alpha_{1}} \otimes \ldots \otimes \phi_{\alpha_{k}},
$$

then the degree $k$ function $f^{(k)}$ will have a branch cut starting at $\phi_{\alpha_{1}}=0$. The discontinuity across this branch cut, denoted by $\Delta_{\phi_{\alpha_{1}}} f^{(k)}$, will also be a pure function, of degree $k-1$. Its symbol is found by clipping the first element off the symbol for $f^{(k)}$ :

$$
\mathcal{S}\left(\Delta_{\phi_{\alpha_{1}}} f^{(k)}\right)=\sum_{\vec{\alpha}} \phi_{\alpha_{2}} \otimes \ldots \otimes \phi_{\alpha_{k}}
$$

In general, taking discontinuities commutes with taking derivatives.

\section{Constraining the three-loop remainder function}

We will now describe a procedure for constraining the form of the remainder function based on a plausible ansatz for its symbol. Our experience with six-point integrals in both four and six dimensions $[54,55,63]$ is that their symbols are always formed of terms with entries drawn from the following set of nine elements,

$$
\left\{u, v, w, 1-u, 1-v, 1-w, y_{u}, y_{v}, y_{w}\right\}
$$

Here we use the notation

$$
y_{u}=\frac{u-z_{+}}{u-z_{-}}, \quad y_{v}=\frac{v-z_{+}}{v-z_{-}}, \quad y_{w}=\frac{w-z_{+}}{w-z_{-}},
$$

where

$$
z_{ \pm}=\frac{1}{2}[-1+u+v+w \pm \sqrt{\Delta}], \quad \Delta=(1-u-v-w)^{2}-4 u v w .
$$

Thus our ansatz for the remainder function at $l$ loops will be the most general symbol of degree $2 l$ that we can make from the above set of nine elements. That is, we assume that the symbol for the remainder function can be factorised in terms of the set (3.1). 
We can also write the cross ratios in terms of ratios of two-brackets of $\mathbb{C P}^{1}$ variables $w_{i}$,

$$
u=\frac{(23)(56)}{(25)(36)}, \quad v=\frac{(34)(61)}{(36)(41)}, \quad w=\frac{(45)(12)}{(41)(52)},
$$

where $(i j)=-(j i)=\epsilon_{a b} w_{i}^{a} w_{j}^{b}$. In these variables, $\Delta$ is a perfect square,

$$
\sqrt{\Delta}= \pm \frac{(12)(34)(56)+(23)(45)(61)}{(14)(25)(36)} .
$$

Taking the positive branch of the square root, and using the Schouten identity for the two-brackets, we have

$$
\begin{aligned}
1-u & =\frac{(35)(26)}{(25)(36)}, & 1-v & =\frac{(46)(31)}{(36)(41)}, \\
y_{u} & =\frac{(23)(46)(15)}{(56)(13)(24)}, & 1-w & =\frac{(51)(42)}{(41)(52)}, \\
y_{v} & =\frac{(61)(24)(35)}{(34)(51)(26)}, & y_{w} & =\frac{(45)(62)(31)}{(12)(35)(46)} .
\end{aligned}
$$

Note that under a cyclic permutation, $w_{i} \rightarrow w_{i+1}$, with indices modulo 6 , the sign of $\sqrt{\Delta}$ flips, $\sqrt{\Delta} \rightarrow-\sqrt{\Delta}$. So the $y$ variables permute as $y_{u} \rightarrow 1 / y_{v} \rightarrow y_{w} \rightarrow 1 / y_{u}$. This inversion will not affect the symmetry properties of the parity-even functions and symbols in which we are interested, which involve even numbers of $y$ variables.

From eqs. (3.4), (3.6) and (3.7) we see that our ansatz is equivalent to saying that the symbol can be factorised in terms of two-brackets $(i j)$ (or equivalently momentum-twistor four-brackets) at the six-point level. (There are 15 two-brackets $(i j)$, but only combinations that are invariant under rescaling of individual $w_{i}$ coordinates are allowed, which reduces the number of independent combinations to the nine exhibited in eqs. (3.4), (3.6) and (3.7).) Note that we can fix a coordinate choice $w_{i}=\left(1, z_{i}\right)$, where these variables coincide with the $z_{i}$ variables of ref. [35], so that our ansatz is also equivalent to assuming that the symbol can be factorised in terms of differences of the $z_{i}$. The form of our ansatz is certainly sufficient at the two-loop level, because the remainder function is explicitly known [6, 7, 32, 33, 35], and its symbol is indeed of this form [35]. In the above variables, it is given by

$$
\begin{aligned}
\mathcal{S}\left(R_{6}^{(2)}\right)= & -\frac{1}{8}\left\{\left[u \otimes(1-u) \otimes \frac{u}{(1-u)^{2}}+2(u \otimes v+v \otimes u) \otimes \frac{w}{1-v}+2 v \otimes \frac{w}{1-v} \otimes u\right] \otimes \frac{u}{1-u}\right. \\
& \left.+\left[u \otimes(1-u) \otimes y_{u} y_{v} y_{w}-2 u \otimes v \otimes y_{w}\right] \otimes y_{u} y_{v} y_{w}\right\}+ \text { permutations },
\end{aligned}
$$

where the sum is over the 6 permutations of $u, v$ and $w$, which correspondingly permute $y_{u}, y_{v}$ and $y_{w}$.

What constraints should the symbol of the remainder function obey?

- It should be integrable, i.e. it should actually be the symbol of a function.

- The first entry in any term of the symbol should be a cross ratio $u, v$ or $w$. The leading entries describe the locations of the discontinuities of the function, which can only originate at $x_{i j}^{2}=0$, as can be seen by considering the unitarity cuts of the amplitude [41]. These points correspond to cuts in $u, v$ or $w$ originating at either 0 or $\infty$. A first entry containing $1-u, y_{u}$, etc., would lead to a discontinuity starting at an unphysical point. 
Within our ansatz for the symbol of the three-loop remainder function, these two constraints are sufficient to show (by explicit enumeration) that the second entry of the symbol can only be drawn from the set $\{u, v, w, 1-u, 1-v, 1-w\}$. This result is consistent with a conjecture of some of the authors of ref. [41]. The second-entry property is of course true for the known two-loop remainder function. We also have the following further conditions on the symbol of the remainder function:

- It should be completely symmetric in the cross ratios $u, v, w$.

- It should be parity even. Because the $y$ variables of eq. (3.2) invert under parity (the exchange of $z_{+}$and $z_{-}$), there should be an even number of $y$ entries in any given term in the symbol.

- It should vanish in the collinear limit. This constraint can be implemented at the level of the symbol as follows. In the limit $w \rightarrow 0$, we find that the $y$ variables behave as

$$
y_{u} \longrightarrow \frac{u}{1-v}, \quad y_{v} \longrightarrow \frac{v}{1-u}, \quad y_{w} \longrightarrow \frac{w(1-u)(1-v)}{(1-u-v)^{2}} .
$$

The collinear limit can be obtained by first taking the $w \rightarrow 0$ limit, factorising the symbol and then taking the limit $v \rightarrow 1-u$. The symbol of the remainder function should then vanish. (A term in the symbol vanishes if at least one of its entries goes smoothly to 1.)

We have analysed the implications of the above constraints up to three loops (i.e. up to symbols of degree six). At one loop we find that there are no symbols obeying all of the above properties. This result is expected, since the remainder function, which vanishes in the collinear limit, starts appearing only at two loops and beyond. At two loops there is a four-parameter family of symbols obeying the constraints that we have outlined. Not surprisingly, it contains the symbol of the two-loop remainder function which is explicitly known [35] and satisfies the above conditions. At three loops we find a 59-parameter space of symbols obeying the constraints outlined above. We would like to impose more constraints to see if we can further restrict the space of possible solutions. We have the following two classes of additional constraints:

- As well as vanishing in the strict collinear limit, the Wilson loop in the near-collinear regime should have an OPE expansion as described in refs. [39-41]. Roughly speaking, this expansion comes about because a Wilson loop can be expanded around the limit where a set of adjacent sides becomes collinear. A scaling parameter $\tau$ measures how close the Wilson loop is to the collinear configuration $(\tau \rightarrow \infty$ corresponds to the strict collinear limit). In terms of this parameter the Wilson loop ${ }^{1}$ should have an expansion of the form

$$
W=\int d n C_{n} e^{-E_{n} \tau}
$$

\footnotetext{
${ }^{1}$ More accurately, one considers the logarithm of a particular finite, conformally invariant ratio of Wilson loops.
} 
Here $n$ is shorthand for the set of labels corresponding to the state being exchanged, $E_{n}$ is the 'energy' of the state (i.e. its eigenvalue under the $\tau$ scaling), and $C_{n}$ corresponds roughly to the probability of emission and absorption of a given state. In principle, a complete knowledge of the set of states labeled by $n$, and expressions for the energies $E_{n}$ and the overlap functions $C_{n}$ entirely fix the remainder function. In fact, armed with a knowledge of only the leading corrections to the energies of the simplest single-particle states, we can predict the leading discontinuity at any loop order. At two loops this information is sufficient to determine the entire symbol [41], because the leading discontinuity is just a single discontinuity, $\Delta_{v} R_{6}^{(2)}$. The discontinuities in the other two cross ratios, $\Delta_{u} R_{6}^{(2)}$ and $\Delta_{w} R_{6}^{(2)}$, are related by symmetry. Using the fact that the first entry of the symbol is either $u, v$ or $w$, and eq. (2.6) for the symbol of the discontinuity, we see that knowing $\Delta_{v} R_{6}^{(2)}$ allows the full two-loop symbol to be reconstructed by appending a $v$ to the front and summing over cyclic permutations. At three loops, the leading corrections to the $E_{n}$ suffice to constrain the double discontinuity, $\Delta_{v} \Delta_{v} R_{6}^{(3)}$. This is a powerful constraint, although it does not uniquely determine the remainder function on its own.

- The remainder function should also obey particular constraints in multi-Regge kinematics [47-50]. In this limit, $u \rightarrow 1$, while $v$ and $w$ vanish in a particular way,

$$
u \longrightarrow 1, \quad \frac{v}{1-u} \longrightarrow x, \quad \frac{w}{1-u} \longrightarrow y .
$$

Here $x$ and $y$ are free parameters. ${ }^{2}$ One must be careful about the branch on which the limit is taken. In fact, the functions we are interested in vanish in this limit in the Euclidean region [51-53] (when all separations $x_{i j}$ are taken to be spacelike) but are non-vanishing and even logarithmically divergent in physical regions for $2 \rightarrow 4$ and $3 \rightarrow 3$ processes [44, 45, 48, 49, 52].

The symbol of the two-loop remainder function is entirely fixed by the OPE [41] to agree with the symbol of the expression found in ref. [35]. The two-loop remainder function has also been shown to obey the multi-Regge constraints [47, 48]. At three loops we find that, of the 59 independent symbols obeying integrability, symmetry and the collinear limit, 26 have no double discontinuity in a given channel. These functions therefore cannot be constrained by the OPE analysis. For the remaining 33 symbols we find that there does exist a unique solution to the OPE constraints (thus adding support to the correctness of the ansatz we have adopted). Thus the OPE fixes 33 of the 59 free parameters of our symbol.

Analysing the multi-Regge limits we find that, of the 26 functions without any double discontinuity, only three are non-vanishing in the multi-Regge kinematics. One has beyondleading-log behaviour (it is proportional to $\log ^{3}(1-u$ ) in the limit (3.11)), and is therefore ruled out. Another parameter is fixed by the known leading-log behaviour, proportional to $\log ^{2}(1-u)[48,49]$. Thus a single parameter is left undetermined in the multi-Regge limit.

\footnotetext{
${ }^{2}$ The variable $y$ introduced in eq. (3.11) should not be confused with the variables $y_{u}, y_{v}$ and $y_{w}$ used for generic kinematics.
} 
This free parameter appears in the next-to-leading log behaviour, but not at the next-tonext-to-leading log level. We will see later that it has to be set to zero for consistency with the all-loop-order prediction concerning $3 \rightarrow 3$ scattering [49].

Having examined the consequences of the above constraints, we find a symbol of the form,

$$
\mathcal{S}\left(R_{6}^{(3)}\right)=\mathcal{S}(X)+\sum_{i=1}^{26} \alpha_{i} \mathcal{S}\left(f_{i}\right) .
$$

The first term, $\mathcal{S}(X)$, is the piece that is fixed by the OPE constraints. The remaining free parameters $\alpha_{i}$ accompany symbols of functions $f_{i}$ which have no double discontinuity. Examining the form of $\mathcal{S}(X)$ we find it can be written in such a way that its final entries are always of the form,

$$
\left\{\frac{u}{1-u}, \frac{v}{1-v}, \frac{w}{1-w}, y_{u}, y_{v}, y_{w}\right\}
$$

Note that this is not in contradiction with the ansatz (3.1), since the entries can always be factorised. Instead it is a more restrictive statement, because only 6 out of the 9 potential variables actually appear in the final entry. This result concerning the restricted structure of the final entries of $\mathcal{S}(X)$ is closely connected to the observations of ref. [38], which suggests that this fact may be related to a supersymmetric formulation of the Wilson loop. Similar restrictions appear [57] in differential equations obeyed [54-56] by integrals related to planar $\mathcal{N}=4$ super-Yang-Mills scattering amplitudes [23]. These observations suggest that the full symbol $\mathcal{S}\left(R_{6}^{(3)}\right)$, not just $\mathcal{S}(X)$, should be of a form in which its final entries are drawn from the list (3.13). Imposing this condition on the final entries of $\mathcal{S}\left(R_{6}^{(3)}\right)$ reduces the number of free parameters to just two. The fact that it is possible to impose this restriction, consistently with the known multi-Regge behaviour, is highly non-trivial.

Finally, let us note that even if we were able to fix the entire symbol and find a function with all the desired analytic properties, vanishing in the collinear limit with the correct OPE behaviour, etc., there would always remain the possibility of adding some amount of the two-loop remainder function multiplied by $\zeta_{2}$, that is,

$$
R_{6}^{(3)} \longrightarrow R_{6}^{(3)}+\gamma \zeta_{2} R_{6}^{(2)},
$$

for some constant $\gamma$. We will see such 'beyond-the-symbol' ambiguities appearing in a particular way in our predictions for the multi-Regge behaviour of the three-loop remainder function.

We will now discuss the OPE analysis in further detail, and then describe the predictions for the three-loop remainder function in the multi-Regge kinematics. We will conclude with a discussion of the conditions on the final entries, and the remaining ambiguities after imposing all our constraints.

\section{OPE constraints}

In order to describe the OPE expansion for a light-like Wilson loop, the authors of ref. [39] introduced a reference square with null sides, denoted by $W_{\text {square }}$ in figure 1 . Two of the sides of the square coincide with two of the sides of the Wilson loop, while the other two 


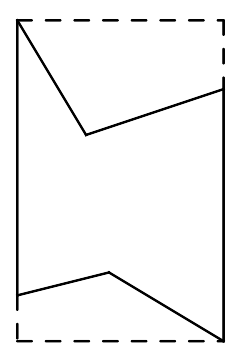

$W_{\text {orig }}$

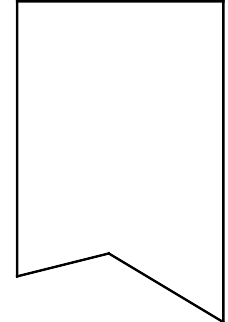

$W_{\text {top }}$

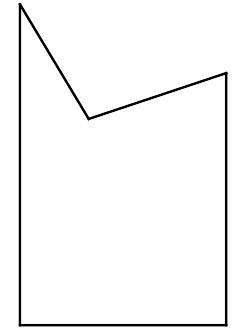

$W_{\text {bottom }}$

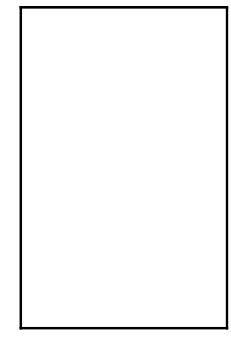

$W_{\text {square }}$

Figure 1. The four different Wilson loops entering the definition of the ratio (4.1). The reference square is shown by the dashed line. The top and bottom Wilson loops are obtained by replacing a sequence of edges by the corresponding part of the square.

sides are formed by finding other null lines that intersect the two previous ones as well as two of the corners of the original loop. One can then consider the finite, conformally invariant quantity made from a ratio of Wilson loops,

$$
r=\log \frac{W_{\text {orig }} W_{\text {square }}}{W_{\text {top }} W_{\text {bottom }}} .
$$

The four different Wilson loops appearing in the ratio are depicted in figure 1 .

Note that at six points, the top and bottom loops are five-sided. The four-sided and five-sided loops appearing in the ratio $r$ are entirely determined by the conformal Ward identity (1.3). Thus knowledge of the quantity $r$ is equivalent to knowing the six-point remainder function.

As described in ref. [41], the Wilson loop, or more precisely the ratio $r$, is expected to have an OPE expansion of the form

$$
r=\int d n C_{n} e^{-E_{n} \tau}
$$

At one loop, the states labelled by $n$ are free single-particle exchanges between the bottom half of the the loop and the top half. Beyond one loop there can be interactions between the particles and the vertical Wilson lines in figure 1, as well as multi-particle exchanges, and so forth. The quantities $C_{n}$ and $E_{n}$ entering the OPE should be expanded in the coupling constant. In principle, to determine $r$ (and hence the remainder function) one needs to know the space of states and the dependence of $C_{n}$ and $E_{n}$ on the coupling.

There is, however, a piece of the remainder function that is completely constrained at $l$ loops, just from knowing the one-loop anomalous dimensions [64] of the single-particle states being exchanged [41]. In the near-collinear limit, one of the cross ratios vanishes, say $v \rightarrow 0$. It vanishes exponentially quickly as $\tau \rightarrow \infty$; that is, $\tau$ is proportional to $\log v$ in this limit. The special piece of the remainder function (or $r$ ) is the leading discontinuity in $v$, which is the repeated $(l-1)$-fold discontinuity $\Delta_{v}^{l-1} r$. This discontinuity can be extracted from the OPE by first Taylor expanding the energies of the intermediate states in the coupling constant,

$$
E_{n}=E_{n}^{(0)}+g^{2} E_{n}^{(1)}+g^{4} E_{n}^{(2)}+\ldots
$$


After Taylor expanding the exponential in eq. (3.10) in $g^{2}$ we find

$$
r=\int d n C_{n} e^{-E_{n}^{(0)} \tau}\left[1-g^{2} \tau E_{n}^{(1)}+g^{4}\left(\frac{1}{2} \tau^{2}\left(E_{n}^{(1)}\right)^{2}-\tau E_{n}^{(2)}\right)+\ldots\right] .
$$

Because $\tau$ is proportional to $\log v$ as $\tau \rightarrow \infty$, the leading discontinuity in $v$ at any loop order is given by the term involving the highest power of $\tau$. This term is always obtained from the one-loop corrections $E_{n}^{(1)}$ to the energies of the simplest single-particle states those states whose overlap functions $C_{n}$ are non-vanishing at order $g^{2}$.

The exchanged states carry other quantum numbers in addition to the energy $E_{n}$. There is a 'momentum' $p$ conjugate to the other scaling ( $\sigma$-scaling) invariance of the square and a discrete label $m$, conjugate to the rotational invariance ( $\phi$-rotation) in the two directions orthogonal to the square. These three invariances of the square can be used to completely parametrise the three variables $u, v$ and $w$ on which $r$ (or the six-point remainder function) depends. Explicitly, the variables $\sigma, \tau$ and $\phi$ are related to $u, v$ and $w$ via

$$
u=\frac{e^{\sigma} \sinh \tau \tanh \tau}{2(\cosh \sigma \cosh \tau+\cos \phi)}, \quad v=\frac{1}{\cosh ^{2} \tau}, \quad w=\frac{e^{-\sigma} \sinh \tau \tanh \tau}{2(\cosh \sigma \cosh \tau+\cos \phi)} .
$$

A more detailed description of the leading discontinuity of $r$ at $l$ loops is then

$$
\begin{aligned}
\Delta_{v}^{l-1} r^{(l)} \propto \frac{(-1)^{l}}{(l-1) !} \int \frac{d p}{2 \pi} e^{-i p \sigma} & \left(\sum_{m=1}^{\infty} \frac{\left[\gamma_{m+2}(p)\right]^{l-1} \cos (m \phi)}{p^{2}+m^{2}}\right. \\
& \left.+\sum_{m=2}^{\infty} \frac{\left[\gamma_{m-2}(p)\right]^{l-1} \cos ((m-2) \phi)}{p^{2}+(m-2)^{2}}\right) \mathcal{C}_{m}(p) \mathcal{F}_{m / 2, p}(\tau)
\end{aligned}
$$

The one-loop anomalous dimensions $\gamma_{m}(p)$ are the energies $E_{n}^{(1)}$ of conformal primary states, and they are given by [64],

$$
\gamma_{m}(p)=\psi\left(\frac{m+i p}{2}\right)+\psi\left(\frac{m-i p}{2}\right)-2 \psi(1)
$$

The explicit formulae for the overlap functions $\mathcal{C}_{m}(p)$ and the conformal blocks $\mathcal{F}_{m / 2, p}(\tau)$, which account for the exchange of conformal descendant states, are given in ref. [41]. The formula (4.6) has been slightly adapted from the corresponding one for two loops [41] by raising the anomalous dimensions $\gamma_{m}(p)$ to the power $l-1$ instead of 1 . This power originates simply from the highest power of $\tau$ at each loop order in eq. (4.4), as in this term the anomalous dimension appears in the exponent accompanied by a factor of $\tau$. In summary, the leading discontinuity in any of the cross ratios (which are all related by the permutation symmetry) is completely predicted by the OPE, in a formula very similar to the two-loop case.

Evaluating the expression (4.6) is quite involved. However, following ref. [41] we can say that it must obey certain differential equations. The differential operators $\mathcal{D}_{ \pm}$of ref. [41] should annihilate any function given by a sum of two towers of conformal blocks. Using 
results from appendix A, one can work out the form of these operators in terms of the cross ratios $u, v$ and $w$ :

$$
\begin{aligned}
\mathcal{D}_{ \pm}=\frac{4}{1-v}[ & -z_{ \pm} u \partial_{u}-(1-v) v \partial_{v}-z_{ \pm} w \partial_{w} \\
& +(1-u) v u \partial_{u} u \partial_{u}+(1-v)^{2} v \partial_{v} v \partial_{v}+(1-w) v w \partial_{w} w \partial_{w} \\
& \left.+(-1+u-v+w)\left((1-v) u \partial_{u} v \partial_{v}-v u \partial_{u} w \partial_{w}+(1-v) v \partial_{v} w \partial_{w}\right)\right] .
\end{aligned}
$$

At any given loop order beyond one loop, the symbol of the remainder function $R_{6}^{(l)}$ is actually equal to the symbol of the Wilson loop ratio $r^{(l)}$. The difference between the two functions comes from additional terms in the expansion of eq. (4.1) in the coupling. For example, in eq. (1.5), $R_{n}$ is a constant for the four- and five-point contributions to $r$, but there are degree two functions (at most) related to $F_{n, 1-\text { loop }}^{\mathrm{WL}}$ that will contribute to the difference between $r$ and the remainder function, when they are multiplied by transcendental constants from higher-order terms in $\gamma_{K}$. These terms drop out of the symbol.

For our three-loop analysis we require that the symbol of the leading (double) discontinuity $\left(\Delta_{v}\right)^{2} R_{6}^{(3)}$ is annihilated by the product of $\mathcal{D}_{+}$and $\mathcal{D}_{-}$,

$$
\mathcal{S}\left(\mathcal{D}_{+} \mathcal{D}_{-} \Delta_{v} \Delta_{v} R_{6}^{(3)}(u, v, w)\right)=0 .
$$

The above is a very general constraint, which should apply to all expressions admitting an OPE expansion of the form described in ref. [39]. Within our specific ansatz it becomes extremely powerful. We find that it fixes 33 out of the 59 coefficients that were undetermined after imposing integrability, symmetry and the collinear limit. The remaining 26 terms have no double discontinuity in any single channel, so they cannot be fixed without supplying additional information.

In ref. [41] the sum (4.6) was performed for the single discontinuity $\Delta_{v} R_{6}^{(2)}$ at two loops, for which only a single power of the anomalous dimensions $\gamma_{m}(p)$ appears. One method used is to compute the discontinuities of the discontinuity. We can perform a similar analysis for the double discontinuities of the double discontinuity at three loops.

At two loops the discontinuity $\Delta_{v} R_{6}^{(2)}$ has further discontinuities of the type $\Delta_{u}, \Delta_{w}$ and $\Delta_{1-v}$. The double discontinuity $\Delta_{w} \Delta_{v} R_{6}^{(2)}$ is a degree two function. When computing the integral (4.6) over $p$ as a sum over residues, it arises from double poles in the $p$ plane for $l=2$. The formula (4.7) for $\gamma_{m}(p)$ contains single poles, with constant residues, at $p=i(m+2 k)$ for non-negative integers $k$. They can combine with single poles at the same locations in the overlap functions $\mathcal{C}_{m}(p)$. For $p=i m$ they can also combine with poles from the $p^{2}+m^{2}$ denominator factor. Double poles give rise to derivatives with respect to $p$, which can hit the exponential $e^{-i p \sigma}$ (the only place $\sigma$ appears) and bring down a factor of $\sigma$. Because $\log w$ is proportional to $\sigma$ in eq. (4.5) as $\sigma \rightarrow+\infty$, the coefficient of the term linear in $\sigma$ yields the discontinuity with respect to $w$.

Similarly, at three loops the double discontinuity of the double discontinuity $\Delta_{w} \Delta_{w} \Delta_{v} \Delta_{v} R_{6}^{(3)}$ arises from triple poles in the $p$ plane in the expression (4.6) for $l=3$, which generate two derivatives with respect to $p$ acting on $e^{-i p \sigma}$. The analysis of appendix B.1 of ref. [41] is almost directly applicable to $\Delta_{w} \Delta_{w} \Delta_{v} \Delta_{v} R_{6}^{(3)}$. However, there is a small 
mismatch due to the factor of $p^{2}+m^{2}$ in the denominator of the terms in the first sum in eq. (4.6). This factor contributes a pole at $p=i m$, which combines with the pole coming from $\mathcal{C}_{m}(p)$ to produce a double-pole contribution to the two-loop expression $\Delta_{w} \Delta_{v} R_{6}^{(2)}$, without requiring a pole from $\gamma_{m+2}(p)$. There are no such contributions for the three-loop expression $\Delta_{w} \Delta_{w} \Delta_{v} \Delta_{v} R_{6}^{(3)}$, because the only triple poles come from combining $\left[\gamma_{m}(p)\right]^{2}$ with $\mathcal{C}_{m}(p)$.

On the other hand, if we could remove the $p^{2}+m^{2}$ factor in the denominator of eq. (4.6), then the same analysis for the two-loop problem would also apply directly at three loops. It is important for this conclusion that the residues of $\gamma_{m}(p)$ are constants, independent of $m$ and $p$. Removing the denominator amounts to acting with the particular second-order operator $\square=-\left(\partial_{\sigma}^{2}+\partial_{\phi}^{2}\right)$ described in ref. [41]. In terms of the cross ratios, using results from appendix $A$, the operator $\square$ is given by

$$
\square=\frac{4 u w}{1-v}\left[u \partial_{u}+w \partial_{w}-(1-u) \partial_{u} u \partial_{u}-(1-w) \partial_{w} w \partial_{w}+(1-u-v-w+2 u w) \partial_{u} \partial_{w}\right] .
$$

We therefore conclude that

$$
\square \Delta_{w} \Delta_{w} \Delta_{v} \Delta_{v} R_{6}^{(3)} \propto \square \Delta_{w} \Delta_{v} R_{6}^{(2)}=\frac{w(1-u+v-w)}{(1-v)(1-w)} .
$$

The second equation can be found by acting with $\square$ on the symbol for the discontinuity of $R_{6}^{(2)}$

$$
\begin{aligned}
\mathcal{S}\left(\Delta_{w} \Delta_{v} R_{6}^{(2)}\right)=-\frac{1}{4}\{ & u \otimes \frac{u v w}{(1-u)(1-v)(1-w)}-(1-w) \otimes \frac{v}{1-v}-(1-v) \otimes \frac{w}{1-w} \\
& \left.-y_{u} \otimes y_{u} y_{v} y_{w}\right\},
\end{aligned}
$$

which is easily extracted from the symbol (3.8) for $R_{6}^{(2)}$. It can also be found by applying $\square$ to the explicit representation for the discontinuity $X_{3}$ found in ref. [41]. (We have not yet fixed the overall normalization of $R_{6}^{(3)}$; we will do this subsequently when we match to the leading-logarithmic behaviour in the multi-Regge limit.) Remarkably, the symbol obtained after imposing the condition (4.9) is perfectly consistent with the condition (4.11), which is a non-trivial check of our analysis.

In conclusion, after imposing the leading OPE constraints we find a solution consistent with our ansatz containing 26 unfixed parameters $\alpha_{i}$,

$$
\mathcal{S}\left(R_{6}^{(3)}\right)=\mathcal{S}(X)+\sum_{i=1}^{26} \alpha_{i} \mathcal{S}\left(f_{i}\right) .
$$

Each of the symbols appearing in the above expression is required to be integrable, and so there do exist functions $X, f_{i}$ with those symbols. The double discontinuities of $X$ and the $f_{i}$ obey

$$
\Delta_{v} \Delta_{v} X \neq 0, \quad \mathcal{D}_{+} \mathcal{D}_{-} \Delta_{v} \Delta_{v} X=0, \quad \mathcal{S}\left(\Delta_{v} \Delta_{v} f_{i}\right)=0 .
$$

Although the symbol for $X$ is one of the central results of this article, it is also rather lengthy. Therefore we do not present it directly in the text. Instead we give it in accompanying Mathematica and plain text files. In these files, a term $a \otimes b \otimes \ldots \otimes f$ is written as 
$\mathrm{SB}[a, b, \ldots, f]$. Using symbol(ic) manipulation programs, it is straightforward to extract information about various limits and discontinuities from the symbol. The next section describes one such limit, multi-Regge kinematics.

\section{$5 \quad$ Predictions for multi-Regge kinematics}

We now analyse our symbol in the multi-Regge limit (3.11), in which $u \rightarrow 1$ while $v$ and $w$ vanish. First we find that in the Euclidean version of this limit, the symbol we have found vanishes, in agreement with observations [51-53] about the consistency of the BDS ansatz in this type of limit. Next we analytically continue to a physical branch, defined by letting $u \rightarrow e^{-2 \pi i} u$. For physical $2 \rightarrow 4$ scattering, $v$ and $w$ remain at their Euclidean values. The imaginary terms on the physical branch that are generated by this transformation of $u$ come from the discontinuity of the function in the $u$ channel in the multi-Regge limit. As mentioned in section 2, the symbol of the discontinuity of a function $f$ in a given channel ( $u$ say) can be found by taking the terms in the original symbol $\mathcal{S}(f)$ with initial entry $u$ and stripping off that entry. The result, after multiplying by $(-2 \pi i)$, is the symbol of the discontinuity $\mathcal{S}\left(\Delta_{u} f\right)$. The real terms for $2 \rightarrow 4$ scattering come from a double discontinuity in the $u$ channel. They are found from $\mathcal{S}\left(\Delta_{u} \Delta_{u} f\right)$, after multiplying by $(2 \pi i)^{2}$. (In principle, there can be contributions to the imaginary and real parts from triple and higher order discontinuities in $u$ as well. However, through three loops there are no such terms.)

The behaviour we expect for the $l$-loop remainder function in the multi-Regge limit in the physical region is

$$
R_{6}^{(l)} \longrightarrow(2 \pi i) \sum_{r=0}^{l-1} \log ^{r}(1-u)\left[g_{r}^{(l)}(x, y)+2 \pi i h_{r}^{(l)}(x, y)\right],
$$

where the logarithmic expansion coefficients $g_{r}^{(l)}$ and $h_{r}^{(l)}$ are functions that depend only on the finite ratios $x$ and $y$ defined in eq. (3.11). It is convenient to change variables to describe these functions. Following ref. [48], we introduce the variables $w, w^{*}$ defined by ${ }^{3}$

$$
x=\frac{1}{(1+w)\left(1+w^{*}\right)}, \quad y=\frac{w w^{*}}{(1+w)\left(1+w^{*}\right)} .
$$

In terms of these variables, the symbols of the functions $g_{r}^{(l)}$ and $h_{r}^{(l)}$ have as their only entries $w, w^{*},(1+w)$, and $\left(1+w^{*}\right)$.

Both $g_{r}^{(l)}$ and $h_{r}^{(l)}$ are invariant under two $Z_{2}$ symmetries:

$$
\text { conjugation : } w \longleftrightarrow w^{*},
$$

which is a reality condition for the case that $w^{*}$ is the complex conjugate of $w$, and

$$
\text { inversion : } w \longleftrightarrow 1 / w, \quad w^{*} \longleftrightarrow 1 / w^{*} .
$$

\footnotetext{
${ }^{3}$ The new variable $w$ in the multi-Regge limit (which is always accompanied by a $w^{*}$ ) should not be confused with the original cross ratio $w$.
} 
The combined operation of inversion and conjugation is the reflection symmetry $x \leftrightarrow y$, which is inherited from the permutation symmetry $v \leftrightarrow w$ for generic kinematics. We also expect the functions $g_{r}^{(l)}$ and $h_{r}^{(l)}$ to be single-valued as $w$ is rotated around the origin of the complex plane. Finally, the functions should vanish for $|w| \rightarrow 0$, which is the collinear limit on top of the Regge limit.

In taking the multi-Regge limit (3.11) of symbols, we note that any symbol containing a $u$ or a $y_{u}$ entry can be discarded, because $u \rightarrow 1$ and $y_{u} \rightarrow 1$ in this limit. We recall the definition of $x$ and $y$ in eq. (3.11). The variables $y_{v}$ and $y_{w}$ go to finite values, $\tilde{y}_{v}$ and $\tilde{y}_{w}$, in the limit:

$$
\begin{aligned}
y_{v} \longrightarrow \tilde{y}_{v} & =\frac{-1-x+y+\sqrt{\tilde{\Delta}}}{-1-x+y-\sqrt{\tilde{\Delta}}}=\frac{1+w^{*}}{1+w}, \\
y_{w} \longrightarrow \tilde{y}_{w} & =\frac{-1+x-y+\sqrt{\tilde{\Delta}}}{-1+x-y-\sqrt{\tilde{\Delta}}}=\frac{(1+w) w^{*}}{w\left(1+w^{*}\right)},
\end{aligned}
$$

where $\tilde{\Delta}=(1-x-y)^{2}-4 x y$ is the limit of $\Delta /(1-u)^{2}$. The relation of $\tilde{y}_{v}$ and $\tilde{y}_{w}$ to the $\left(w, w^{*}\right)$ variables can be found with the aid of formulae in appendix B.

The symbols $\mathcal{S}\left(g_{r}^{(l)}\right)$ and $\mathcal{S}\left(h_{r}^{(l)}\right)$ do not fix the functions $g_{r}^{(l)}$ and $h_{r}^{(l)}$ uniquely. One can always add transcendental constants such as $\zeta_{2}$, multiplied by lower transcendentality functions which vanish in the symbol. However, the above symmetries, eqs. (5.3) and (5.4), and analytic properties around $w=0$, greatly restrict the form of such potential ambiguities. In particular there are no such functions of degree 0 or 1 obeying these constraints.

Before describing the three-loop predictions, we recall $[47,48]$ the corresponding expansion (5.1) at two loops, as obtained from the formula of ref. [35],

$$
\begin{aligned}
g_{1}^{(2)}\left(w, w^{*}\right)= & \frac{1}{4} \log |1+w|^{2} \log \frac{|1+w|^{2}}{|w|^{2}} \\
g_{0}^{(2)}\left(w, w^{*}\right)= & \frac{1}{4} \log |w|^{2} \log ^{2}|1+w|^{2}-\frac{1}{6} \log ^{3}|1+w|^{2}+\frac{1}{2} \log |w|^{2}\left[\operatorname{Li}_{2}(-w)+\operatorname{Li}_{2}\left(-w^{*}\right)\right] \\
& -\operatorname{Li}_{3}(-w)-\operatorname{Li}_{3}\left(-w^{*}\right) .
\end{aligned}
$$

It is not always the case that $w^{*}$ is the complex conjugate of $w$. (That only happens if $\sqrt{\tilde{\Delta}}$ is imaginary.) In the general case, $|w|^{2}$ is just a shorthand for $w w^{*}$, and $|1+w|^{2}$ is a shorthand for $(1+w)\left(1+w^{*}\right)$.

The functions controlling the real parts depend on whether the scattering is $2 \rightarrow 4$ or $3 \rightarrow 3$. In $2 \rightarrow 4$ scattering, the multi-Regge limit has vanishing real part at two loops [47],

$$
\begin{aligned}
& h_{1}^{(2)}\left(w, w^{*}\right)=0, \\
& h_{0}^{(2)}\left(w, w^{*}\right)=0 .
\end{aligned}
$$

In the case of $3 \rightarrow 3$ scattering, $v$ and $w$ have to be analytically continued to the opposite sign from their Euclidean values [49]; that is,

$$
u \rightarrow|u| e^{2 \pi i}, \quad v \rightarrow|v| e^{\pi i}, \quad w \rightarrow|w| e^{\pi i} .
$$


In fact, the remainder function for $3 \rightarrow 3$ scattering can be derived from the $2 \rightarrow 4$ case by the simple substitution

$$
\log (1-u) \longrightarrow \log (u-1)-i \pi
$$

followed by complex conjugation [49].

Whereas the function $g_{1}^{(2)}$ in eq. (5.7) is manifestly invariant under both conjugation and inversion symmetries, $g_{0}^{(2)}$ in eq. (5.8) only has manifest invariance under $w \leftrightarrow w^{*}$. On the other hand, this form makes clear that $g_{0}^{(2)}$ vanishes as $|w| \rightarrow 0$, and also that it acquires no phase as $w$ is rotated around the origin of the complex plane. The latter property is obvious for $|w|<1$ and true by inversion symmetry for $|w|>1$. Simple polylogarithm identities can be used to demonstrate the $w$ inversion symmetry. In fact, assuming maximal transcendentality, the functions $g_{1}^{(2)}$ and $g_{0}^{(2)}$, of degree 2 and 3 respectively, can be fixed uniquely, just by knowing the symbol of the two-loop remainder function and imposing these requirements. The uniqueness holds because the symbol fixes the functions up to constants like $\zeta_{3}$ or $\zeta_{2}$, multiplied by functions of corresponding lower degree, and there are no functions with degree 0 or 1 obeying the constraints.

At three loops we find that in the multi-Regge limit, the symbol $\mathcal{S}(X)$ has the form of the symbol of the right-hand side of eq. (5.1) for $l=3$, with the leading divergence being a double logarithmic one. We also find that in this limit, all but three of the $\mathcal{S}\left(f_{i}\right)$ vanish. We will call the functions with non-vanishing symbols in the limit $f_{24}, f_{25}, f_{26}$. We find that one symbol, $\mathcal{S}\left(f_{26}\right)$, has a triple logarithmic divergence in the multi-Regge limit, which is one logarithm beyond the known degree of divergence. Therefore the coefficient $\alpha_{26}$ must vanish. The symbol $\mathcal{S}(X)$ contributes to the double logarithmic divergence exactly what is required to match the symbol of the leading-log prediction [48]. We find that $\mathcal{S}\left(f_{25}\right)$ also contributes a double logarithmic divergence (different in form from that of $\mathcal{S}(X)$ ). Hence we deduce that its coefficient $\alpha_{25}$ must vanish, so that it does not spoil the agreement with the leading-log prediction. The final symbol $\mathcal{S}\left(f_{24}\right)$ then contributes to the next-to-leading$\log$ term (i.e. to $\mathcal{S}\left(g_{1}^{(3)}\right)$ ) but not to the next-to-next-to-leading one (i.e. not to $\mathcal{S}\left(g_{0}^{(3)}\right)$ ). Because it is the only arbitrary coefficient from the expression (4.13) that survives in the multi-Regge limit (after imposing the constraints we have just discussed), we give it a new name, $\alpha_{24}=c$.

Now we describe our predictions for the multi-Regge limit, after imposing the conditions,

$$
\alpha_{24}=c, \quad \alpha_{25}=0, \quad \alpha_{26}=0 .
$$

We find (as described above) that the symbol of $g_{2}^{(3)}$ agrees precisely with the symbol of the coefficient of the $\log ^{2}(1-u)$ term predicted in ref. [48], namely

$$
\mathcal{S}\left(g_{2}^{(3)}\right)=\frac{1}{32}\left(2 x \otimes x \otimes y+3 x \otimes y \otimes x y-x \otimes \tilde{y}_{w} \otimes \tilde{y}_{v} \tilde{y}_{w}\right)+(x \longleftrightarrow y) .
$$

We have adjusted the overall normalization of $X$ so that this term in the multi-Regge limit agrees with ref. [48]. This normalization is based on the loop expansion parameter $a=g^{2} N_{c} /\left(8 \pi^{2}\right)$ and eq. (1.6). 
When written in terms of the $w, w^{*}$ variables, the symbol (5.14) can be seen to be the symbol of the following function,

$$
g_{2}^{(3)}\left(w, w^{*}\right)=\frac{1}{8} g_{0}^{(2)}\left(w, w^{*}\right)-\frac{1}{32} \log |1+w|^{2} \log \frac{|1+w|^{2}}{|w|^{2}} \log \frac{|1+w|^{4}}{|w|^{2}},
$$

exactly as predicted in ref. [48]. Just as in the two-loop case, this degree 3 function is uniquely determined by its symbol, because there are no suitable degree 1 or 0 functions one could add. Also, we find from the double $u$ discontinuity that the real part at leadinglog level vanishes,

$$
h_{2}^{(3)}\left(w, w^{*}\right)=0
$$

as expected.

We also have predictions for the symbols of $g_{1}^{(3)}, g_{0}^{(3)}$ and $h_{0}^{(3)}$ (and their corresponding functions) which are new. The function $h_{1}^{(3)}$ for $2 \rightarrow 4$ kinematics was predicted in ref. [48], and we obtain the same function. Remarkably, all these functions can be expressed in terms of classical polylogarithms.

As the transcendental degree increases, it becomes more difficult to write the result in a form that is simultaneously invariant under inversion of $w$, and manifestly has good behaviour as $|w| \rightarrow 0$. We choose to express the functions in a form where the $|w| \rightarrow 0$ behaviour is manifest. (Alternate forms with manifest inversion symmetry can be found in appendix C.) At the next-to-leading-log level, we find

$$
\begin{aligned}
g_{1}^{(3)}\left(w, w^{*}\right)= & \frac{1}{8}\left\{\log |w|^{2}\left[\operatorname{Li}_{3}\left(\frac{w}{1+w}\right)+\operatorname{Li}_{3}\left(\frac{w^{*}}{1+w^{*}}\right)\right]\right. \\
& +\left(5 \log |1+w|^{2}-2 \log |w|^{2}\right)\left[\operatorname{Li}_{3}(-w)+\operatorname{Li}_{3}\left(-w^{*}\right)\right] \\
& -\frac{3}{2} \log |w|^{2} \log \frac{|1+w|^{4}}{|w|^{2}}\left[\operatorname{Li}_{2}(-w)+\operatorname{Li}_{2}\left(-w^{*}\right)\right] \\
& -\frac{1}{12} \log ^{2}|1+w|^{2}\left[\log |w|^{2}\left(\log |w|^{2}+2 \log |1+w|^{2}\right)-10 \log ^{2} \frac{|1+w|^{2}}{|w|^{2}}\right] \\
& \left.+\frac{1}{2} \log |w|^{2} \log \frac{|1+w|^{2}}{|w|^{2}} \log (1+w) \log \left(1+w^{*}\right)-2 \zeta_{3} \log |1+w|^{2}\right\} \\
& +\left(\frac{5}{2}+\gamma^{\prime}\right) \zeta_{2} g_{1}^{(2)}\left(w, w^{*}\right)+c g_{1}^{a} .
\end{aligned}
$$

For this degree-four function there are only two constants to determine. The first one, $\gamma^{\prime}$, corresponds to the freedom to add the two-loop remainder function, multiplied by $\zeta_{2}$, to the three-loop remainder function, as in eq. (3.14). The second constant, $c$, is the remaining ambiguity at the level of the symbol. It multiplies the function,

$$
\begin{aligned}
g_{1}^{a}\left(w, w^{*}\right)= & 4 \log \frac{|1+w|^{2}}{|w|^{2}}\left[\operatorname{Li}_{3}(-w)+\operatorname{Li}_{3}\left(-w^{*}\right)\right]-4 \log |w|^{2}\left[\operatorname{Li}_{3}\left(\frac{w}{1+w}\right)+\operatorname{Li}_{3}\left(\frac{w^{*}}{1+w^{*}}\right)\right] \\
& +2\left[\operatorname{Li}_{2}(-w)-\operatorname{Li}_{2}\left(-w^{*}\right)+\log |w|^{2} \log \frac{1+w}{1+w^{*}}\right]\left[\operatorname{Li}_{2}(-w)-\operatorname{Li}_{2}\left(-w^{*}\right)\right] \\
& +\frac{1}{6} \log ^{3}|1+w|^{2}\left(\log |1+w|^{2}+2 \log |w|^{2}\right) \\
& -2 \log |w|^{2} \log |1+w|^{2} \log (1+w) \log \left(1+w^{*}\right)+8 \zeta_{3} \log |1+w|^{2} .
\end{aligned}
$$


We will see later that this function does not enter, i.e. that $c=0$, if we impose consistency with the all-loop-order prediction for $3 \rightarrow 3$ kinematics [49]. Also, in section 6 we will see that this function can also be excluded by imposing an additional constraint on the form of the final entries in the symbol of $R_{6}^{(3)}$.

We rule out additional constants multiplying lower-degree transcendental functions in eq. (5.17) by first assuming that potential functions must be built from logarithms and (at high enough degree) polylogarithms containing the same arguments found in the leadingtranscendentality (symbol-level) terms, namely $\log w, \log (1+w), \operatorname{Li}_{m}(-w), \operatorname{Li}_{m}(w /(1+$ $w)$ ) and $\operatorname{Li}_{m}(1 /(1+w))$ (for $m=2,3$ these polylogarithms are not all independent). After enumerating such functions, we impose the four constraints discussed above: the conjugation and inversion symmetries; vanishing of the function in the (collinear-Regge) $|w| \rightarrow 0$ limit; and absence of a phase as $w$ is rotated around the origin of the complex plane. These constraints rule out functions of degree 0 or 1 . The unique function of degree 2 obeying these conditions is $g_{1}^{(2)}\left(w, w^{*}\right)$. If we had omitted the final-entry condition, for example, we could have added a term proportional to

$$
\zeta_{2} \log \left(\frac{1+w}{1+w^{*}}\right) \log \left(\frac{(1+w) w^{*}}{\left(1+w^{*}\right) w}\right) .
$$

This term has both symmetries and vanishes as $|w| \rightarrow 0$; in fact, it is the unique term at degree two that satisfies the other three constraints but violates the phase condition.

The degree-three function controlling the real part at next-to-leading-log level, $h_{1}^{(3)}$, can be found from the multi-Regge limit of the double $u$ discontinuity. (There is an overall factor of $1 / 2$ associated with the fact that the symbol of $\log ^{2} u$ is $2 u \otimes u$.) We find

$$
\mathcal{S}\left(h_{1}^{(3)}\right)=\mathcal{S}\left(g_{2}^{(3)}\right)-\frac{1}{8}[x \otimes y \otimes y+y \otimes x \otimes y+y \otimes y \otimes x+(x \longleftrightarrow y)],
$$

which integrates to

$$
h_{1}^{(3)}\left(w, w^{*}\right)=g_{2}^{(3)}\left(w, w^{*}\right)+\frac{1}{16} \log |1+w|^{2} \log \frac{|1+w|^{2}}{|w|^{2}} \log \frac{|1+w|^{4}}{|w|^{2}} .
$$

This result agrees with that found in ref. [48].

Moving on to next-to-next-to-leading-log level, we find the degree-five function controlling the imaginary part,

$$
\begin{gathered}
g_{0}^{(3)}\left(w, w^{*}\right)=-\frac{1}{32}\left\{-60\left[2\left(\operatorname{Li}_{5}(-w)+\operatorname{Li}_{5}\left(-w^{*}\right)\right)-\log |w|^{2}\left(\operatorname{Li}_{4}(-w)+\operatorname{Li}_{4}\left(-w^{*}\right)\right)\right]\right. \\
+12\left[2 \left(\operatorname{Li}_{5}\left(\frac{w}{1+w}\right)+\operatorname{Li}_{5}\left(\frac{1}{1+w}\right)+\frac{1}{24} \log w \log ^{4}(1+w)\right.\right. \\
\left.+\operatorname{Li}_{5}\left(\frac{w^{*}}{1+w^{*}}\right)+\operatorname{Li}_{5}\left(\frac{1}{1+w^{*}}\right)+\frac{1}{24} \log w^{*} \log ^{4}\left(1+w^{*}\right)\right) \\
+\log \frac{|1+w|^{2}}{|w|^{2}}\left(\operatorname{Li}_{4}\left(\frac{w}{1+w}\right)+\operatorname{Li}_{4}\left(\frac{w^{*}}{1+w^{*}}\right)\right) \\
+\log |1+w|^{2}\left(\operatorname{Li}_{4}\left(\frac{1}{1+w}\right)-\frac{1}{6} \log w \log ^{3}(1+w)\right. \\
\left.\left.+\operatorname{Li}_{4}\left(\frac{1}{1+w^{*}}\right)-\frac{1}{6} \log w^{*} \log ^{3}\left(1+w^{*}\right)\right)\right]
\end{gathered}
$$




$$
\left.\begin{array}{l}
-2\left(5\left(\log ^{2}|w|^{2}-\log ^{2}|1+w|^{2}\right)+6 \log |w|^{2} \log |1+w|^{2}\right)\left(\operatorname{Li}_{3}(-w)+\operatorname{Li}_{3}\left(-w^{*}\right)\right) \\
-2 \log |w|^{2} \log \frac{|1+w|^{4}}{|w|^{2}}\left(\operatorname{Li}_{3}\left(\frac{w}{1+w}\right)+\operatorname{Li}_{3}\left(\frac{w^{*}}{1+w^{*}}\right)\right) \\
-6 \log |w|^{2} \log |1+w|^{2} \log \frac{|1+w|^{2}}{|w|^{2}}\left(\operatorname{Li}_{2}(-w)+\operatorname{Li}_{2}\left(-w^{*}\right)\right) \\
+\frac{5}{3} \log ^{5}|1+w|^{2}-\frac{5}{2} \log |w|^{2} \log ^{4}|1+w|^{2}+\frac{4}{3} \log ^{2}|w|^{2} \log ^{3}|1+w|^{2} \\
-\log |w|^{2} \log ^{2}(1+w) \log ^{2}\left(1+w^{*}\right)-2 \log ^{3}|1+w|^{2} \log (1+w) \log \left(1+w^{*}\right) \\
+\zeta_{2} \log |w|^{2} \log |1+w|^{2}\left(\log |w|^{2}-3 \log |1+w|^{2}\right)+4 \zeta_{3} \log |w|^{2} \log |1+w|^{2}-48 \zeta_{5}
\end{array}\right\}
$$

Note that although $\operatorname{Li}_{m}(1 /(1+w))$ has logarithmic branch-cut behaviour near $w=0$, the combination

$$
\operatorname{Li}_{m}\left(\frac{1}{1+w}\right)-\frac{(-1)^{m}}{(m-1) !} \log w \log ^{m-1}(1+w)
$$

is well-behaved. This property can be verified inductively by differentiating with respect to $w$ and using

$$
\frac{d}{d w} \operatorname{Li}_{m}\left(\frac{1}{1+w}\right)=-\frac{1}{1+w} \operatorname{Li}_{m-1}\left(\frac{1}{1+w}\right) .
$$

After using the combination (5.23) in $g_{0}^{(3)}$, there are no other bare $\log w$ terms; they all come along with a $\log w^{*}$ to form $\log |w|^{2}$. Note that for $m=3$ one can use an identity to eliminate $\operatorname{Li}_{3}(1 /(1+w))$ in favor of $\operatorname{Li}_{m}(-w)$ and $\operatorname{Li}_{3}(w /(1+w))$, but there is no such identity for $m>3$.

As was the case for $g_{1}^{(3)}$, all possible constraints will be satisfied by a function proportional to the two-loop remainder function, multiplied by $\zeta_{2}$. This accounts for the term proportional to $g_{0}^{(2)}\left(w, w^{*}\right)$. In addition, we can multiply the two-loop leading-log multiRegge coefficient $g_{1}^{(2)}$ by $\zeta_{3}$, to get something with the right transcendental degree and satisfying the above constraints. Presumably its coefficient, $d_{1}$, can be fixed by additional beyond-the-symbol information. Finally, there is another degree-three function satisfying all the constraints we imposed, with a coefficient $d_{2}$ which we expect to be fixed in a similar fashion. This purely-logarithmic degree-three function is a linear combination of the next-to-leading-log two-loop function $g_{0}^{(2)}$ and the leading-log three-loop function $g_{2}^{(3)}$, as in eq. (5.15).

The real part at next-to-next-to-leading-log level is given by the degree-four function,

$$
\begin{aligned}
& h_{0}^{(3)}\left(w, w^{*}\right)=\frac{1}{16}\left\{-\left(3 \log |1+w|^{2}-2 \log |w|^{2}\right)\left[\operatorname{Li}_{3}(-w)+\operatorname{Li}_{3}\left(-w^{*}\right)\right]\right. \\
& +\log |w|^{2}\left[\operatorname{Li}_{3}\left(\frac{w}{1+w}\right)+\operatorname{Li}_{3}\left(\frac{w^{*}}{1+w^{*}}\right)\right]+\frac{1}{2} \log |w|^{2} \log \frac{|1+w|^{4}}{|w|^{2}}\left[\operatorname{Li}_{2}(-w)+\operatorname{Li}_{2}\left(-w^{*}\right)\right] \\
& -\frac{1}{2} \log ^{4}|1+w|^{2}+\frac{5}{6} \log ^{3}|1+w|^{2} \log |w|^{2}-\frac{1}{4} \log ^{2}|1+w|^{2} \log ^{2}|w|^{2} \\
& \left.+\frac{1}{2} \log |w|^{2} \log \frac{|1+w|^{2}}{|w|^{2}} \log (1+w) \log \left(1+w^{*}\right)-2 \zeta_{3} \log |1+w|^{2}\right\}+\zeta_{2} \gamma^{\prime \prime \prime} g_{1}^{(2)}
\end{aligned}
$$


As was the case for eq. (5.17), the term containing an explicit $\zeta_{3}$ in eq. (5.25) is fixed using the symmetries and the vanishing of $h_{0}^{(3)}$ as $|w| \rightarrow 0$. There is an arbitrary constant $\gamma^{\prime \prime \prime}$ multiplying $g_{1}^{(2)}$, but we will see shortly how to fix it.

In ref. [46], the scattering amplitude in the multi-Regge limit was expressed as a sum of Regge pole and Mandelstam cut contributions. By using this representation, general formulae were obtained for the multi-Regge limit of the remainder function in both $2 \rightarrow 4$ and $3 \rightarrow 3$ kinematics, in terms of a real function $f(\omega ; x, y)$ characterizing the partial waves entering the Mandelstam cut,

$$
\begin{array}{ll}
\exp \left[R_{6}+i \pi \delta\right]=\cos \pi \omega_{a b}+i \int_{-i \infty}^{i \infty} \frac{d \omega}{2 \pi i} f(\omega ; x, y) e^{-i \pi \omega}|1-u|^{-\omega} & (2 \rightarrow 4) \\
\exp \left[R_{6}-i \pi \delta\right]=\cos \pi \omega_{a b}-i \int_{-i \infty}^{i \infty} \frac{d \omega}{2 \pi i} f(\omega ; x, y)|1-u|^{-\omega} & (3 \rightarrow 3) .
\end{array}
$$

Here

$$
\begin{aligned}
\exp \left[R_{6}\right] & =1+a^{2} R_{6}^{(2)}+a^{3} R_{6}^{(3)}+\ldots, \\
\delta & =-\frac{\gamma_{K}}{8} \log \frac{|1+w|^{4}}{|w|^{2}}, \\
\omega_{a b} & =\frac{\gamma_{K}}{8} \log |w|^{2}
\end{aligned}
$$

and the cusp anomalous dimension $\gamma_{K}$ is given by

$$
\gamma_{K}=4 a-4 \zeta_{2} a^{2}+22 \zeta_{4} a^{3}+\ldots
$$

in terms of the coupling constant $a=g^{2} N_{c} /\left(8 \pi^{2}\right)$. Note that the quantity appearing in eqs. (5.26) and (5.27) is the ratio of the full amplitude (or Wilson loop) to the BDS ansatz, which according to our conventions (see eqs. (1.5) and (1.6)) is the exponential of the remainder function. The phase $\delta$ comes from the behavior of the BDS ansatz in the multi-Regge limit, while $\omega_{a b}$ is derived from the Regge-pole contribution.

Remarkably, the second term in eq. (5.27), containing $f(\omega ; x, y)$, drops out when we take the real part of the equation, leading to the all-loop-order relation for $3 \rightarrow 3$ kinematics [49],

$$
\operatorname{Re}\left\{\exp \left[R_{6}-i \pi \delta\right]\right\}=\cos \pi \omega_{a b} \quad(3 \rightarrow 3)
$$

The factor of $e^{-i \pi \omega}$ inside the integral in eq. (5.26) prevents an analogously simple relation from holding for $2 \rightarrow 4$ scattering.

By using the results given above for the functions $g_{r}^{(l)}$ and $h_{r}^{(l)}$ through $l=3$, we can easily test eq. (5.32) at the three-loop level. We assemble the exponential of the remainder function, $\exp \left[R_{6}\right]$ in eq. (5.28), using eq. (5.1) for $2 \rightarrow 4$ kinematics. Then we apply the substitution (5.12), followed by complex conjugation, to convert the result into the one for $3 \rightarrow 3$ kinematics. Dressing the result with $e^{-i \pi \delta}$, taking the real part, and setting $h_{1}^{(2)}=h_{0}^{(2)}=h_{2}^{(3)}=0$, we find the following relations, at orders $a^{2}, a^{3} \log (u-1)$ and $a^{3}$, 
respectively:

$$
\begin{aligned}
& g_{1}^{(2)}=\frac{\left(\gamma_{K}^{(1)}\right)^{2}}{64} \log |1+w|^{2} \log \frac{|1+w|^{2}}{|w|^{2}}, \\
& h_{1}^{(3)}=g_{2}^{(3)}+\frac{\gamma_{K}^{(1)}}{16} \log \frac{|1+w|^{4}}{|w|^{2}} g_{1}^{(2)}, \\
& h_{0}^{(3)}=\frac{1}{2} g_{1}^{(3)}+\frac{\gamma_{K}^{(1)}}{16} \log \frac{|1+w|^{4}}{|w|^{2}} g_{0}^{(2)}-\frac{\gamma_{K}^{(1)} \gamma_{K}^{(2)}}{64} \log |1+w|^{2} \log \frac{|1+w|^{2}}{|w|^{2}},
\end{aligned}
$$

where $\gamma_{K}^{(1)}=4, \gamma_{K}^{(2)}=-4 \zeta_{2}$. Equation (5.33) is equivalent to eq. (5.7), and eq. (5.34) is equivalent to eq. (5.21). Inserting the above values for $h_{0}^{(3)}, g_{1}^{(3)}$ and $g_{0}^{(2)}$ into eq. (5.35), we see that eq. (5.32) is satisfied precisely, through three loops - but only if we set $c=0$ in eq. (5.17) for $g_{1}^{(3)}$. In addition we must fix the constant $\gamma^{\prime \prime \prime}$ in eq. (5.25) for $h_{0}^{(3)}$ to the value,

$$
\gamma^{\prime \prime \prime}=\frac{9}{4}+\frac{\gamma^{\prime}}{2}
$$

The imaginary part $g_{1}^{(3)}$ for $2 \rightarrow 4$ kinematics contributes to the real part of $R_{6}^{(3)}$ for $3 \rightarrow 3$ kinematics because of the substitution (5.12) and the fact that $g_{1}^{(3)}$ is multiplied by $\log (1-u)$ in eq. (5.1). In fact, the only function that does not enter eq. (5.32) is the degree-five function $g_{0}^{(3)}$, because it is from the imaginary part and has no $\log (1-u)$ multiplying it. Hence eq. (5.32) is a powerful check on our results.

The $c=0$ constraint imposed by eq. (5.32) also arises from considering restrictions on the final entry of the symbol, as we shall do in the next section.

\section{Constraints on the final entry of the symbol}

We have shown that within the specific ansatz (3.1) we were able to write the symbol of the three-loop remainder function in the form

$$
\mathcal{S}\left(R_{6}^{(3)}\right)=\mathcal{S}(X)+\sum_{i=1}^{24} \alpha_{i} \mathcal{S}\left(f_{i}\right) .
$$

There are 24 unfixed parameters $\alpha_{i}$, after imposing all of the constraints we have outlined, including the constraints coming from the multi-Regge limit (5.13). Moreover, by examining the symbol $\mathcal{S}(X)$ we find that it is possible to write it so that the final entries are drawn from the following set,

$$
\left\{\frac{u}{1-u}, \frac{v}{1-v}, \frac{w}{1-w}, y_{u}, y_{v}, y_{w}\right\}
$$

The same restriction is true for the symbol of the full remainder function $R_{6}^{(2)}$ at two loops, given in eq. (3.8). As mentioned above, it has been suggested [38] that this fact is related to a supersymmetric formulation of the Wilson loop; and similar restrictions appear [57] in differential equations [54-56] for integrals related to scattering amplitudes [23]. It is reasonable to think that the full symbol $\mathcal{S}\left(R_{6}^{(3)}\right)$ should obey this condition, including 
the ambiguities $\mathcal{S}\left(f_{i}\right)$. In fact, it is possible to impose this condition on the remaining ambiguities, leaving just two free parameters,

$$
\mathcal{S}\left(R_{6}^{(3)}\right)=\mathcal{S}(X)+\alpha_{1} \mathcal{S}\left(f_{1}\right)+\alpha_{2} \mathcal{S}\left(f_{2}\right)
$$

The fact that this form for the symbol is consistent with the known Regge behaviour is highly non-trivial. Indeed, one can adopt the constraint on the final entries from the beginning. In this case, after imposing the OPE constraints, the triple-log in the multiRegge limit vanishes automatically, and the leading-log contribution $g_{2}^{(3)}$ is uniquely fixed to agree with the prediction of refs. [49, 50]. Finally, the single remaining free parameter in the multi-Regge limit (which appears in the function $g_{1}^{(3)}$ in eq. (5.17)) is fixed,

$$
c=0
$$

leaving a completely unambiguous prediction for the symbol of $g_{1}^{(3)}$ in this limit (the symbol for $g_{0}^{(3)}$ was already fixed unambiguously). It is reassuring that the same vanishing value for $c$ is also dictated by the relation (5.32) for the multi-Regge limit for $3 \rightarrow 3$ kinematics.

The symbol $\mathcal{S}\left(f_{1}\right)$ is extremely simple: It is entirely composed from the entries $\{u, v, w, 1-u, 1-v, 1-w\}$; the square-root containing $y$ variables in eq. (3.1) do not appear in $\mathcal{S}\left(f_{1}\right)$. This property makes it straightforward to find an explicit function $f_{1}$, which has the symbol $\mathcal{S}\left(f_{1}\right)$. The function can be written in the form,

$$
f_{1}(u, v, w)=h(u) h(v)+h(u) h(w)+h(v) h(w)+k(u)+k(v)+k(w) .
$$

Here the single-variable functions $h$ and $k$ are given by

$$
\begin{aligned}
h(u)= & \frac{1}{3} \log ^{3} u+\log u \operatorname{Li}_{2}(1-u)-\operatorname{Li}_{3}(1-u)-2 \operatorname{Li}_{3}(1-1 / u), \\
k(u)= & -\log ^{3} u H_{3}+\frac{3}{2} \log ^{2} u\left(H_{4}-H_{2,2}-4 H_{3,1}\right) \\
& -\log u\left(H_{2,3}-6 H_{4,1}+H_{2,1,2}+6 H_{2,2,1}+18 H_{3,1,1}\right) \\
& +3 H_{2,4}+4 H_{3,3}+3 H_{4,2}+H_{2,1,3}-H_{2,2,2}-2 H_{2,3,1} \\
& -2 H_{3,1,2}+9 H_{4,1,1}-2 H_{2,1,2,1}-9 H_{2,2,1,1}-24 H_{3,1,1,1} .
\end{aligned}
$$

The arguments of the harmonic polylogarithms appearing in $k(u)$ are all $(1-u)$ and have been suppressed to save space. A subscript $m$ stands for $m-1$ zero entries followed by a single 1 entry [58]; so for example $H_{3,1,2}=H_{0,0,1,1,0,1}(1-u)$.

The function $f_{1}$ above has been chosen so that it obeys

$$
\partial_{u} f_{1}=\frac{1}{u(1-u)}(\text { pure function }) .
$$

The fact that taking the derivative yields a pure function with the particular $1 /(u(1-u))$ prefactor is the functional consequence of the final-entry condition on the symbol. The function $f_{1}$ is real-valued in the Euclidean region but does not vanish in the collinear limit. It only vanishes up to terms involving explicit appearances of $\zeta_{2}\left(\pi^{2}\right)$ and $\zeta_{3}$, which is what 
is guaranteed by the form of its symbol. In fact, already at the $\zeta_{2}$ level we find that $f_{1}$ is divergent in this limit,

$$
\begin{aligned}
\lim _{w \rightarrow 0} f_{1}=\zeta_{2}[ & \log w\left(\frac{1}{2} \log u \log ^{2}(1-u)+\log u \operatorname{Li}_{2}(u)+2 \log (1-u) \operatorname{Li}_{2}(u)-3 \operatorname{Li}_{3}(u)+3 H_{2,1}(u)\right) \\
& + \text { finite }]+\zeta_{3}[\ldots]+\zeta_{4}[\ldots]+\zeta_{5}[\ldots]+\zeta_{2} \zeta_{3}[\ldots]
\end{aligned}
$$

In fact there exists no degree 4 function with a symbol within our ansatz, and also obeying the property (6.8), which could be used to remove this divergence in the collinear limit. This fact suggests that if we insist on preserving the functional consequence of the final entry condition (6.8), beyond the level of the symbol, then there will be additional constraints on the parameter $\alpha_{1}$ when completing the symbol $\mathcal{S}\left(R_{6}^{(3)}\right)$ to a genuine function.

The function $f_{2}$ is intermediate in complexity between $f_{1}$ and $X$. Its symbol contains terms with up to two $y$-variable entries, while the symbol for $X$ has terms with four $y$ variable entries. Files containing the symbols for $f_{1}, f_{2}$ and $X$, as well as the symbols of the functions characterizing the multi-Regge limit (which for $c=0$ come entirely from $X$ ), are provided as auxiliary material for this paper.

We leave to later work an explicit construction of functions associated with the other symbols, particularly $\mathcal{S}(X)$ and $\mathcal{S}\left(f_{2}\right)$. However, we can already say some things about the full three-loop remainder function. In particular, for any values of $\alpha_{1}$ and $\alpha_{2}$, it is impossible to represent its symbol by a function given in terms of (products of) only single-variable harmonic polylogarithms $H_{\vec{w}}(x)$, whose weight vectors $\vec{w}$ contain only the entries 0 and 1. As a corollary, it is not possible to represent the symbol by a function given purely in terms of the classical polylog functions $\operatorname{Li}_{n}(x)$, for any choices of $x$. This result can be obtained by performing symmetry operations similar to those described in ref. [35]. It is sufficient, and a bit simpler, to test not the full symbol, but a particular piece of it. We take the double discontinuity in $w$, and then set $w \rightarrow 0$, using the relations (3.9). This symbol is given by

$$
\begin{aligned}
&\left.\mathcal{S}\left(\Delta_{w} \Delta_{w} X\right)\right|_{w \rightarrow 0}=\frac{1}{8}\left\{u \otimes u \otimes\left[-(1-u) \otimes \frac{u v}{(1-u)(1-u-v)}+v \otimes \frac{1-v}{1-u-v}\right]\right. \\
&+u \otimes(1-u) \otimes[ \frac{1-u}{(1-u-v)^{2}} \otimes \frac{u v}{(1-u)(1-u-v)}+\frac{u}{(1-u-v)^{2}} \otimes \frac{(1-u)^{2}(1-v)}{(1-u-v)^{3}} \\
&\left.\quad+v(1-u-v) \otimes \frac{(1-u)(1-v)}{(1-u-v)^{2}}\right] \\
&+u \otimes v \otimes\left[-2 \frac{(1-u)(1-v)}{1-u-v} \otimes \frac{u v}{(1-u-v)^{2}}+u(1-u-v) \otimes \frac{1-v}{1-u-v}\right. \\
&\left.\left.+v(1-u-v) \otimes \frac{1-u}{1-u-v}\right]\right\}+(u \longleftrightarrow v) .
\end{aligned}
$$

We replace each term of the form $a \otimes b \otimes c \otimes d$ in this expression with the following antisymmetrisation [35]:

$$
[(a \otimes b \otimes c \otimes d-(c \leftrightarrow d))-(a \leftrightarrow b)]-[(a, b) \leftrightarrow(c, d)] .
$$


We find that eq. (6.10) is nonvanishing under this operation. The symbol of a degree four function constructed solely from products of single-variable harmonic polylogarithms with labels 0 and 1 (which includes all $\operatorname{Li}_{n}$ functions) vanishes under this operation. Hence $\left(\Delta_{w} \Delta_{w} X\right)_{w \rightarrow 0}$, and also $X$ itself, must include functions beyond this class.

We have also performed a similar test on the full degree six function. Given a degree six symbol which is a sum of terms of the form $a \otimes b \otimes c \otimes d \otimes e \otimes f$, we replace each term with the following antisymmetrisation,

$$
[((a \otimes b \otimes c \otimes d \otimes e \otimes f-(e \leftrightarrow f))-(c \leftrightarrow d))-(a \leftrightarrow b)]-[(a, b) \leftrightarrow(e, f)] .
$$

The symbol of a degree six function constructed solely from products of single-variable harmonic polylogarithms with labels 0 and 1 vanishes under this operation. We find that $\mathcal{S}(X)$ does not vanish under this operation, so again we conclude that $X$ must include functions beyond this class.

\section{Conclusions and outlook}

In this paper we determined the symbol of the remainder function for the three-loop hexagon Wilson loop, or six-point MHV scattering amplitude, in planar $\mathcal{N}=4$ superYang-Mills theory, up to a few undetermined constants. There are 26 such constants in a more general ansatz, but this number drops to just two if a final-entry restriction is imposed on the symbol. The OPE expansion, as analysed in refs. [39-41], provides a powerful constraint for this problem, which is straightforward to implement with the aid of symbols. In particular, we uniquely determined the symbol $\mathcal{S}(X)$ for the part of the three-loop remainder function that has a leading discontinuity.

In the multi-Regge limit, all but one of the symbol-level constants drop out (all of them drop out when we impose the final-entry restriction). In this limit, we are able to complete the symbols for the coefficients in the logarithmic expansion into full analytic functions of degree 3, 4 and 5. These functions depend on two variables, yet they can all be expressed in terms of classical polylogarithms. Three of these functions represent new predictions for the behaviour of the amplitude in the multi-Regge limit. We found confirmation of the final-entry restriction by testing an all-order relation for the remainder function in multi-Regge kinematics for $3 \rightarrow 3$ scattering.

Although only classical polylogarithms appear in the multi-Regge limit, we could use our symbol to show that for more generic kinematics, the three-loop remainder function cannot be expressed solely in terms of classical polylogarithms. Clearly it is an important task to complete the terms in this symbol into full functions. For $f_{1}$, one of the two terms that we could not fix using the leading discontinuity (assuming the final-entry restriction), we were able to accomplish this task. This function is particularly simple due to the fact that its symbol does not depend on the $y$ variables, but only on $\{u, v, w, 1-u, 1-v, 1-w\}$. It factorises into single-variable functions constructed out of harmonic polylogarithms.

The next simplest component is $f_{2}$. It is only quadratic in the $y$ variables, so in some sense it is not much more complicated than the two-loop remainder function, although it is of degree six instead of four. The most complicated term is $X$, which is quartic in the 
$y$ variables. However, we are optimistic that a relatively compact representation for it, as well as $f_{2}$, may be possible to find. We are also encouraged by the fact that the collinear limits of $f_{1}$, which diverge beyond the symbol level, cannot be repaired within functions obeying the final-entry restriction. This fact suggests that the repair may come instead through the collinear behaviour of $X$ and $f_{2}$, which could in turn fix one or both of the arbitrary constants $\alpha_{1}$ and $\alpha_{2}$. It would be remarkable if the three-loop remainder function could be completely determined, or perhaps up to a single ambiguity associated with the two-loop remainder function, without ever directly evaluating a single loop integral, for either a Wilson loop or a scattering amplitude.

\section{Acknowledgments}

We would like to thank Harald Dorn and Thomas Gehrmann for useful discussions. J.M.H. is grateful to the KITP, Santa Barbara, for hospitality while this work was carried out. This work was supported in part by the National Science Foundation under Grant No. PHY0551164, and by the US Department of Energy under contract DE-AC02-76SF00515.

\section{A Change of variables between $u, v, w$ and $\tau, \sigma, \phi$}

In this appendix we provide handy equations for relating various differential operators in term of the variables $\tau, \sigma$ and $\phi$ to those in terms of the cross ratio variables $u, v$ and $w$. From eq. (4.5) we have the auxiliary relations

$$
\begin{aligned}
\frac{1-u-v-w}{1-v} & =\frac{\cos \phi}{\cosh \sigma \cosh \tau+\cos \phi}, & \frac{4 u v w}{(1-v)^{2}} & =\frac{1}{(\cosh \sigma \cosh \tau+\cos \phi)^{2}}, \\
\frac{\sqrt{\Delta}}{1-v} & =\frac{i \sin \phi}{\cosh \sigma \cosh \tau+\cos \phi}, & \tanh \tau & =\sqrt{1-v} .
\end{aligned}
$$

Using these relations, it is simple to show that

$$
\begin{aligned}
& \frac{1}{u} \frac{\partial u}{\partial \tau}=\frac{1}{w} \frac{\partial w}{\partial \tau}=\frac{1-u+v-w}{\sqrt{1-v}}, \quad \frac{1}{v} \frac{\partial v}{\partial \tau}=-2 \sqrt{1-v}, \\
& \frac{1}{u} \frac{\partial u}{\partial \sigma}=\frac{1-u-v+w}{1-v}, \quad \frac{1}{w} \frac{\partial w}{\partial \sigma}=-\frac{1+u-v-w}{1-v}, \quad \frac{\partial v}{\partial \sigma}=0, \\
& \frac{1}{u} \frac{\partial u}{\partial \phi}=\frac{1}{w} \frac{\partial w}{\partial \phi}=\frac{1}{i} \frac{\sqrt{\Delta}}{1-v}, \quad \frac{\partial v}{\partial \phi}=0 .
\end{aligned}
$$

Then the operators differentiating with respect to $\tau, \sigma$ and $\phi$ are

$$
\begin{aligned}
& \partial_{\tau}=\frac{1}{\sqrt{1-v}}\left[-2(1-v) v \partial_{v}+(1-u+v-w)\left(u \partial_{u}+w \partial_{w}\right)\right], \\
& \partial_{\sigma}=\frac{1}{1-v}\left[(1-u-v+w) u \partial_{u}-(1+u-v-w) w \partial_{w}\right], \\
& \partial_{\phi}=\frac{\sqrt{\Delta}}{i(1-v)}\left(u \partial_{u}+w \partial_{w}\right) .
\end{aligned}
$$


Inserting these expressions into the form for $\mathcal{D}_{ \pm}$given in ref. [41],

$$
\mathcal{D}_{ \pm}=\partial_{\tau}^{2}+2 \operatorname{coth}(2 \tau) \partial_{\tau}+\operatorname{sech}^{2} \tau \partial_{\sigma}^{2}+\partial_{\phi}\left(\partial_{\phi} \mp 2 i\right),
$$

it is straightforward to obtain the form in terms of $u, v$ and $w$ given in eq. (4.8). Similarly, the operator $\square=-\left(\partial_{\sigma}^{2}+\partial_{\phi}^{2}\right)$ is found to have the form given in eq. (4.10).

\section{B Change of variables between $u, v, w$ and $y_{u}, y_{v}, y_{w}$}

Although the $y$ variables are constructed using square roots of the original cross ratios $u$, $v$ and $w$, the cross ratios themselves are rational combinations of the variables $y_{u}, y_{v}$ and $y_{w}$. The explicit relations are,

$$
\begin{aligned}
u & =\frac{y_{u}\left(1-y_{v}\right)\left(1-y_{w}\right)}{\left(1-y_{w} y_{u}\right)\left(1-y_{u} y_{v}\right)}, \quad v=\frac{y_{v}\left(1-y_{w}\right)\left(1-y_{u}\right)}{\left(1-y_{u} y_{v}\right)\left(1-y_{v} y_{w}\right)}, \quad w=\frac{y_{w}\left(1-y_{u}\right)\left(1-y_{v}\right)}{\left(1-y_{v} y_{w}\right)\left(1-y_{w} y_{u}\right)}, \\
1-u & =\frac{\left(1-y_{u}\right)\left(1-y_{u} y_{v} y_{w}\right)}{\left(1-y_{w} y_{u}\right)\left(1-y_{u} y_{v}\right)}, \quad 1-v=\frac{\left(1-y_{v}\right)\left(1-y_{u} y_{v} y_{w}\right)}{\left(1-y_{u} y_{v}\right)\left(1-y_{v} y_{w}\right)}, \\
1-w & =\frac{\left(1-y_{w}\right)\left(1-y_{u} y_{v} y_{w}\right)}{\left(1-y_{v} y_{w}\right)\left(1-y_{w} y_{u}\right)}, \quad \sqrt{\Delta}=\frac{\left(1-y_{u}\right)\left(1-y_{v}\right)\left(1-y_{w}\right)\left(1-y_{u} y_{v} y_{w}\right)}{\left(1-y_{u} y_{v}\right)\left(1-y_{v} y_{w}\right)\left(1-y_{w} y_{u}\right)},
\end{aligned}
$$

where we have picked a particular branch of $\sqrt{\Delta}$. These formulas are also useful in the multi-Regge limit. The limit (3.11) corresponds to taking $y_{u} \rightarrow 1, y_{v} \rightarrow \tilde{y}_{v}, y_{w} \rightarrow \tilde{y}_{w}$. We find in the limit,

$$
x=\frac{\tilde{y}_{v}\left(1-\tilde{y}_{w}\right)^{2}}{\left(1-\tilde{y}_{v} \tilde{y}_{w}\right)^{2}}, \quad y=\frac{\tilde{y}_{w}\left(1-\tilde{y}_{v}\right)^{2}}{\left(1-\tilde{y}_{v} \tilde{y}_{w}\right)^{2}}, \quad \sqrt{\tilde{\Delta}}=\frac{\left(1-\tilde{y}_{v}\right)\left(1-\tilde{y}_{w}\right)}{1-\tilde{y}_{v} \tilde{y}_{w}} .
$$

The variables $w$ and $w^{*}$ used in the multi-Regge limit, defined in eq. (5.2), are also rational combinations of $\tilde{y}_{v}$ and $\tilde{y}_{w}$ :

$$
w=\frac{1-\tilde{y}_{v}}{\tilde{y}_{v}\left(1-\tilde{y}_{w}\right)}, \quad w^{*}=\frac{\tilde{y}_{w}\left(1-\tilde{y}_{v}\right)}{1-\tilde{y}_{w}} .
$$

Inverting these equations gives the expressions for $\tilde{y}_{v}$ and $\tilde{y}_{w}$ in terms of $w$ and $w^{*}$ given in eqs. (5.5) and (5.6).

\section{Inversion-symmetric form of functions describing the multi-Regge limit}

Here we write the three-loop functions $g_{r}^{(3)}$ and $h_{r}^{(3)}$ in a form that makes the $w$ inversion and $w \leftrightarrow w^{*}$ symmetries manifest. To do so, we introduce functions $\hat{g}_{r}^{(l)}\left(w, w^{*}\right)$ and $\hat{h}_{r}^{(l)}\left(w, w^{*}\right)$ such that the sum over images under the two symmetries yields the full functions:

$$
\begin{aligned}
& g_{r}^{(l)}\left(w, w^{*}\right)=\hat{g}_{r}^{(l)}\left(w, w^{*}\right)+\hat{g}_{r}^{(l)}\left(w^{*}, w\right)+\hat{g}_{r}^{(l)}\left(1 / w, 1 / w^{*}\right)+\hat{g}_{r}^{(l)}\left(1 / w^{*}, 1 / w\right), \\
& h_{r}^{(l)}\left(w, w^{*}\right)=\hat{h}_{r}^{(l)}\left(w, w^{*}\right)+\hat{h}_{r}^{(l)}\left(w^{*}, w\right)+\hat{h}_{r}^{(l)}\left(1 / w, 1 / w^{*}\right)+\hat{h}_{r}^{(l)}\left(1 / w^{*}, 1 / w\right) .
\end{aligned}
$$


We find that

$$
\begin{aligned}
\hat{g}_{2}^{(3)}\left(w, w^{*}\right)=-\frac{1}{32}[ & 2 \operatorname{Li}_{3}(-w)-\log |w|^{2} \operatorname{Li}_{2}(-w) \\
& \left.-\frac{1}{12} \log ^{2}|1+w|^{2}\left(\log |1+w|^{2}-9 \log \frac{|1+w|^{2}}{|w|^{2}}\right)\right],
\end{aligned}
$$

which agrees with eq. (5.15) and with ref. [48] after the use of a few polylogarithm identities. Similarly, $h_{1}^{(3)}$ can be written symmetrically using

$$
\begin{aligned}
\hat{h}_{1}^{(3)}\left(w, w^{*}\right)=-\frac{1}{32}[ & 2 \operatorname{Li}_{3}(-w)-\log |w|^{2} \operatorname{Li}_{2}(-w) \\
& \left.-\frac{1}{12} \log ^{2}|1+w|^{2}\left(\log |1+w|^{2}+3 \log \frac{|1+w|^{2}}{|w|^{2}}\right)\right] .
\end{aligned}
$$

The new functions found in this paper are $g_{1}^{(3)}, g_{0}^{(3)}$ and $h_{0}^{(3)}$. For $g_{1}^{(3)}$ the symmetric form uses

$$
\begin{aligned}
\hat{g}_{1}^{(3)}\left(w, w^{*}\right)=-\frac{1}{32}\{ \\
\quad 4\left(5 \log |1+w|^{2}-2 \log |w|^{2}\right) \operatorname{Li}_{3}\left(\frac{1}{1+w}\right)+3 \log |w|^{2} \log \frac{|1+w|^{4}}{|w|^{2}} \operatorname{Li}_{2}(-w) \\
\quad+\frac{3}{16}\left[\log ^{4}|w|^{2}+8 \log ^{2}|w|^{2} \log \left(1+w^{*}\right) \log \frac{1+w}{w}+2 \log |w|^{2} \log \frac{w}{w^{*}} \log ^{2} \frac{(1+w)^{2}}{w}\right] \\
\left.\quad-5 \log \frac{|1+w|^{2}}{|w|^{2}} \log |1+w|^{2} \log (1+w) \log \frac{1+w}{w}+\frac{3}{2} \zeta_{2} \log ^{2}|w|^{2}-8 \zeta_{3} \log |1+w|^{2}\right\} \\
+\frac{\zeta_{2}}{4} \gamma^{\prime} g_{1}^{(2)}\left(w, w^{*}\right)+c \hat{g}_{1}^{a},
\end{aligned}
$$

The constant $c$ multiplies the function,

$$
\begin{aligned}
\hat{g}_{1}^{a}= & -4 \log |1+w|^{2} \operatorname{Li}_{3}\left(\frac{w}{1+w}\right)+\operatorname{Li}_{2}(-w)\left[\operatorname{Li}_{2}(-w)-\operatorname{Li}_{2}\left(-w^{*}\right)+\log |w|^{2} \log \frac{1+w}{1+w^{*}}\right] \\
& +\frac{1}{24} \log ^{4}|1+w|^{2}-\frac{1}{4} \log |w|^{2} \log ^{2}|1+w|^{2} \log \frac{|1+w|^{2}}{|w|^{2}} \\
& +\frac{1}{2} \log |w|^{2} \log w \log (1+w) \log \frac{1+w}{w^{*}} \\
& +\frac{1}{3} \log |1+w|^{2} \log ^{2}(1+w)(2 \log (1+w)-3 \log w) \\
& -\zeta_{2} \log |1+w|^{2} \log \frac{|1+w|^{2}}{|w|^{2}}+4 \zeta_{3} \log |1+w|^{2} .
\end{aligned}
$$

Recall that $c=0$ if we impose either the all-loop-order prediction for $3 \rightarrow 3$ scattering [49], or an additional constraint on the form of the final entries in the symbol of $R_{6}^{(3)}$, as described in section 6 . 
The function entering the symmetric form for $g_{0}^{(3)}$ is

$$
\begin{aligned}
\hat{g}_{0}^{(3)}\left(w, w^{*}\right)=-\frac{1}{32}\{ \\
\quad-30\left(2 \operatorname{Li}_{5}(-w)-\log |w|^{2} \operatorname{Li}_{4}(-w)\right)+12\left(2 \operatorname{Li}_{5}\left(\frac{1}{1+w}\right)+\log |1+w|^{2} \operatorname{Li}_{4}\left(\frac{1}{1+w}\right)\right) \\
+\left(-6 \log ^{2}|w|^{2}-4 \log |w|^{2} \log |1+w|^{2}+5 \log ^{2}|1+w|^{2}\right) \operatorname{Li}_{3}(-w) \\
+\log |w|^{2} \log \frac{|1+w|^{4}}{|w|^{2}} \operatorname{Li}_{3}\left(\frac{1}{1+w}\right)-3 \log |w|^{2} \log |1+w|^{2} \log \frac{|1+w|^{2}}{|w|^{2}} \operatorname{Li}_{2}(-w) \\
-\frac{1}{48} \log \frac{|1+w|^{4}}{|w|^{2}}\left(\log ^{4}|1+w|^{2}+\log ^{4} \frac{|1+w|^{2}}{|w|^{2}}-9 \log ^{2}|1+w|^{2} \log ^{2} \frac{|1+w|^{2}}{|w|^{2}}\right) \\
+\frac{1}{32} \log \frac{w}{w^{*}} \log |w|^{2} \log ^{2}|1+w|^{2} \\
-\frac{1}{32} \log \frac{1+w}{1+w^{*}}\left(\log ^{2}|w|^{2}+2 \log |1+w|^{2} \log \frac{|1+w|^{2}}{|w|^{2}}\right) \\
\left.\quad \times\left(2 \log \frac{w}{w^{*}} \log _{\mid 1}|1+w|^{2}-\log \frac{1+w}{1+w^{*}} \log \frac{|1+w|^{4}}{|w|^{2}}\right)+\frac{1}{2} \zeta_{3} \log ^{2}|w|^{2}-12 \zeta_{5}\right\} \\
+\frac{\zeta_{3}}{4} d_{1} g_{1}^{(2)}\left(w, w^{*}\right)+\frac{\zeta_{2}}{4} \gamma^{\prime \prime} g_{0}^{(2)}\left(w, w^{*}\right)+\frac{\zeta_{2}}{4} d_{2} \log |1+w|^{2} \log \frac{|1+w|^{2}}{|w|^{2}} \log \frac{|1+w|^{4}}{|w|^{2}} .
\end{aligned}
$$

Finally, the function needed to write $h_{0}^{(3)}$ in a symmetric form is

$$
\begin{aligned}
\hat{h}_{0}^{(3)}\left(w, w^{*}\right)=\frac{1}{128}\{ \\
\quad 8\left(3 \log |1+w|^{2}-2 \log |w|^{2}\right) \operatorname{Li}_{3}\left(\frac{1}{1+w}\right)+2 \log |w|^{2} \log \frac{|1+w|^{4}}{|w|^{2}} \operatorname{Li}_{2}(-w) \\
+\frac{1}{8}\left[\log ^{4}|w|^{2}+8 \log ^{2}|w|^{2} \log \left(1+w^{*}\right) \log \frac{1+w}{w}+2 \log |w|^{2} \log \frac{w}{w^{*}} \log ^{2} \frac{(1+w)^{2}}{w}\right] \\
\left.-6 \log \frac{|1+w|^{2}}{|w|^{2}} \log |1+w|^{2} \log (1+w) \log \frac{1+w}{w}+\zeta_{2} \log ^{2}|w|^{2}-16 \zeta_{3} \log |1+w|^{2}\right\} \\
+\frac{\zeta_{2}}{4}\left(\frac{3}{4}+\gamma^{\prime \prime \prime}\right) g_{1}^{(2)}\left(w, w^{*}\right) .
\end{aligned}
$$

Polylogarithm identities are required to go between the manifestly symmetric forms of the functions $g_{r}^{(3)}\left(w, w^{*}\right)$ and $h_{r}^{(3)}\left(w, w^{*}\right)$ given in this appendix, and the forms (5.15), (5.17), (5.18), (5.21), (5.22) and (5.25) given in the main text, which have good behaviour as $w \rightarrow 0$.

Open Access. This article is distributed under the terms of the Creative Commons Attribution Noncommercial License which permits any noncommercial use, distribution, and reproduction in any medium, provided the original author(s) and source are credited.

\section{References}

[1] L.F. Alday and J.M. Maldacena, Gluon scattering amplitudes at strong coupling, JHEP 06 (2007) 064 [arXiv:0705.0303] [INSPIRE]. 
[2] J. Drummond, G. Korchemsky and E. Sokatchev, Conformal properties of four-gluon planar amplitudes and Wilson loops, Nucl. Phys. B 795 (2008) 385 [arXiv:0707.0243] [INSPIRE].

[3] A. Brandhuber, P. Heslop and G. Travaglini, $M H V$ amplitudes in $\mathcal{N}=4$ super Yang-Mills and Wilson loops, Nucl. Phys. B 794 (2008) 231 [arXiv:0707.1153] [INSPIRE].

[4] J. Drummond, J. Henn, G. Korchemsky and E. Sokatchev, On planar gluon amplitudes/Wilson loops duality, Nucl. Phys. B 795 (2008) 52 [arXiv:0709.2368] [INSPIRE].

[5] J. Drummond, J. Henn, G. Korchemsky and E. Sokatchev, The hexagon Wilson loop and the BDS ansatz for the six-gluon amplitude, Phys. Lett. B 662 (2008) 456 [arXiv:0712.4138] [INSPIRE].

[6] Z. Bern, L. Dixon, D. Kosower, R. Roiban, M. Spradlin, et al., The two-loop six-gluon MHV amplitude in maximally supersymmetric Yang-Mills theory, Phys. Rev. D 78 (2008) 045007 [arXiv: 0803.1465] [INSPIRE].

[7] J. Drummond, J. Henn, G. Korchemsky and E. Sokatchev, Hexagon Wilson loop = six-gluon MHV amplitude, Nucl. Phys. B 815 (2009) 142 [arXiv: 0803.1466] [INSPIRE].

[8] N. Berkovits and J. Maldacena, Fermionic T-duality, dual superconformal symmetry and the amplitude/Wilson loop connection, JHEP 09 (2008) 062 [arXiv: 0807.3196] [INSPIRE].

[9] J. Drummond, J. Henn, G. Korchemsky and E. Sokatchev, Conformal Ward identities for Wilson loops and a test of the duality with gluon amplitudes, Nucl. Phys. B 826 (2010) 337 [arXiv:0712.1223] [INSPIRE].

[10] I. Korchemskaya and G. Korchemsky, On lightlike Wilson loops, Phys. Lett. B 287 (1992) 169 [inSPIRE].

[11] N. Beisert, B. Eden and M. Staudacher, Transcendentality and crossing, J. Stat. Mech. 0701 (2007) P01021 [hep-th/0610251] [INSPIRE].

[12] Z. Bern, L.J. Dixon and V.A. Smirnov, Iteration of planar amplitudes in maximally supersymmetric Yang-Mills theory at three loops and beyond, Phys. Rev. D 72 (2005) 085001 [hep-th/0505205] [INSPIRE].

[13] J. Drummond, J. Henn, V. Smirnov and E. Sokatchev, Magic identities for conformal four-point integrals, JHEP 01 (2007) 064 [hep-th/0607160] [INSPIRE].

[14] Z. Bern, M. Czakon, L.J. Dixon, D.A. Kosower and V.A. Smirnov, The four-loop planar amplitude and cusp anomalous dimension in maximally supersymmetric Yang-Mills theory, Phys. Rev. D 75 (2007) 085010 [hep-th/0610248] [INSPIRE].

[15] Z. Bern, J. Carrasco, H. Johansson and D. Kosower, Maximally supersymmetric planar Yang-Mills amplitudes at five loops, Phys. Rev. D 76 (2007) 125020 [arXiv:0705.1864] [INSPIRE].

[16] N. Beisert, R. Ricci, A.A. Tseytlin and M. Wolf, Dual superconformal symmetry from $A d S_{5} \times S^{5}$ superstring integrability, Phys. Rev. D 78 (2008) 126004 [arXiv:0807.3228] [INSPIRE].

[17] J. Drummond, J. Henn, G. Korchemsky and E. Sokatchev, Dual superconformal symmetry of scattering amplitudes in $\mathcal{N}=4$ super-Yang-Mills theory, Nucl. Phys. B 828 (2010) 317 [arXiv: 0807.1095] [INSPIRE].

[18] J.M. Drummond, J.M. Henn and J. Plefka, Yangian symmetry of scattering amplitudes in $\mathcal{N}=4$ super Yang-Mills theory, JHEP 05 (2009) 046 [arXiv:0902.2987] [INSPIRE]. 
[19] L.F. Alday, J.M. Henn, J. Plefka and T. Schuster, Scattering into the fifth dimension of $\mathcal{N}=4$ super Yang-Mills, JHEP 01 (2010) 077 [arXiv:0908.0684] [INSPIRE].

[20] Z. Bern, J.J. Carrasco, T. Dennen, Y.-t. Huang and H. Ita, Generalized unitarity and six-dimensional helicity, Phys. Rev. D 83 (2011) 085022 [arXiv: 1010.0494] [INSPIRE].

[21] S. Caron-Huot and D. O'Connell, Spinor helicity and dual conformal symmetry in ten dimensions, JHEP 08 (2011) 014 [arXiv: 1010.5487] [INSPIRE].

[22] T. Dennen and Y.-t. Huang, Dual conformal properties of six-dimensional maximal super Yang-Mills amplitudes, JHEP 01 (2011) 140 [arXiv:1010.5874] [INSPIRE].

[23] N. Arkani-Hamed, J.L. Bourjaily, F. Cachazo, S. Caron-Huot and J. Trnka, The all-loop integrand for scattering amplitudes in planar $\mathcal{N}=4$ SYM, JHEP 01 (2011) 041 [arXiv: 1008.2958] [INSPIRE].

[24] L.J. Dixon, Gluon scattering in $\mathcal{N}=4$ super-Yang-Mills theory from weak to strong coupling, PoS (RAD COR 2007) 056 [arXiv:0803.2475] [INSPIRE].

[25] L.F. Alday and R. Roiban, Scattering amplitudes, Wilson loops and the string/gauge theory correspondence, Phys. Rept. 468 (2008) 153 [arXiv:0807.1889] [INSPIRE].

[26] J. Henn, Duality between Wilson loops and gluon amplitudes, Fortsch. Phys. 57 (2009) 729 [arXiv: 0903.0522] [INSPIRE].

[27] J.M. Henn, Dual conformal symmetry at loop level: massive regularization, J. Phys. A 44 (2011) 454011 [arXiv:1103.1016] [inSPIRE].

[28] T. Adamo, M. Bullimore, L. Mason and D. Skinner, Scattering amplitudes and Wilson loops in twistor space, J. Phys. A 44 (2011) 454008 [arXiv:1104.2890] [inSPIRE].

[29] J. Drummond, Hidden simplicity of gauge theory amplitudes, Class. Quant. Grav. 27 (2010) 214001 [arXiv: 1010.2418] [INSPIRE].

[30] J. Drummond, Review of AdS/CFT integrability, Chapter V.2: Dual superconformal symmetry, arXiv: 1012.4002 [INSPIRE].

[31] T. Bargheer, N. Beisert and F. Loebbert, Exact superconformal and Yangian symmetry of scattering amplitudes, J. Phys. A 44 (2011) 454012 [arXiv:1104.0700] [INSPIRE].

[32] V. Del Duca, C. Duhr and V.A. Smirnov, An analytic result for the two-loop hexagon Wilson loop in $\mathcal{N}=4 S Y M, J H E P 03$ (2010) 099 [arXiv:0911.5332] [INSPIRE].

[33] V. Del Duca, C. Duhr and V.A. Smirnov, The two-loop hexagon Wilson loop in $\mathcal{N}=4 S Y M$, JHEP 05 (2010) 084 [arXiv: 1003.1702] [INSPIRE].

[34] V. Del Duca, C. Duhr and V.A. Smirnov, A two-loop octagon Wilson loop in $\mathcal{N}=4 S Y M$, JHEP 09 (2010) 015 [arXiv: 1006.4127] [INSPIRE].

[35] A.B. Goncharov, M. Spradlin, C. Vergu and A. Volovich, Classical polylogarithms for amplitudes and Wilson loops, Phys. Rev. Lett. 105 (2010) 151605 [arXiv:1006.5703] [INSPIRE].

[36] P. Heslop and V.V. Khoze, Analytic results for MHV Wilson loops, JHEP 11 (2010) 035 [arXiv: 1007.1805] [INSPIRE].

[37] A.B. Goncharov, A simple construction of Grassmannian polylogarithms, arXiv:0908.2238

[38] S. Caron-Huot, Superconformal symmetry and two-loop amplitudes in planar $\mathcal{N}=4$ super Yang-Mills, arXiv:1105.5606 [INSPIRE]. 
[39] L.F. Alday, D. Gaiotto, J. Maldacena, A. Sever and P. Vieira, An operator product expansion for polygonal null Wilson loops, JHEP 04 (2011) 088 [arXiv: 1006.2788] [INSPIRE].

[40] D. Gaiotto, J. Maldacena, A. Sever and P. Vieira, Bootstrapping null polygon Wilson loops, JHEP 03 (2011) 092 [arXiv: 1010.5009] [INSPIRE].

[41] D. Gaiotto, J. Maldacena, A. Sever and P. Vieira, Pulling the straps of polygons, arXiv: 1102.0062 [INSPIRE].

[42] A. Sever and P. Vieira, Multichannel conformal blocks for polygon Wilson loops, arXiv: 1105.5748 [INSPIRE].

[43] A. Sever, P. Vieira and T. Wang, OPE for super loops, arXiv:1108.1575 [INSPIRE].

[44] J. Bartels, L. Lipatov and A. Sabio Vera, BFKL pomeron, Reggeized gluons and Bern-Dixon-Smirnov amplitudes, Phys. Rev. D 80 (2009) 045002 [arXiv:0802.2065] [INSPIRE].

[45] J. Bartels, L. Lipatov and A. Sabio Vera, $\mathcal{N}=4$ supersymmetric Yang-Mills scattering amplitudes at high energies: The Regge cut contribution, Eur. Phys. J. C 65 (2010) 587 [arXiv:0807.0894] [INSPIRE].

[46] L. Lipatov, Analytic properties of high energy production amplitudes in $\mathcal{N}=4$ SUSY, arXiv: 1008.1015 [INSPIRE].

[47] L. Lipatov and A. Prygarin, Mandelstam cuts and light-like Wilson loops in $\mathcal{N}=4$ SUSY, Phys. Rev. D 83 (2011) 045020 [arXiv: 1008.1016] [INSPIRE].

[48] L. Lipatov and A. Prygarin, BFKL approach and six-particle $M H V$ amplitude in $\mathcal{N}=4$ super Yang-Mills, Phys. Rev. D 83 (2011) 125001 [arXiv:1011.2673] [INSPIRE].

[49] J. Bartels, L. Lipatov and A. Prygarin, $M H V$ amplitude for $3 \rightarrow 3$ gluon scattering in Regge limit, arXiv:1012.3178 [INSPIRE].

[50] J. Bartels, L. Lipatov and A. Prygarin, Collinear and Regge behavior of $2 \rightarrow 4 M H V$ amplitude in $\mathcal{N}=4$ super Yang-Mills theory, arXiv:1104.4709 [INSPIRE].

[51] R.C. Brower, H. Nastase, H.J. Schnitzer and C.-I. Tan, Implications of multi-Regge limits for the Bern-Dixon-Smirnov conjecture, Nucl. Phys. B 814 (2009) 293 [arXiv:0801.3891] [INSPIRE].

[52] R.C. Brower, H. Nastase, H.J. Schnitzer and C.-I. Tan, Analyticity for multi-Regge limits of the Bern-Dixon-Smirnov amplitudes, Nucl. Phys. B 822 (2009) 301 [arXiv:0809.1632] [INSPIRE].

[53] V. Del Duca, C. Duhr and E. Glover, Iterated amplitudes in the high-energy limit, JHEP 12 (2008) 097 [arXiv: 0809.1822] [INSPIRE].

[54] J.M. Drummond, J.M. Henn and J. Trnka, New differential equations for on-shell loop integrals, JHEP 04 (2011) 083 [arXiv: 1010.3679] [INSPIRE].

[55] L.J. Dixon, J.M. Drummond and J.M. Henn, The one-loop six-dimensional hexagon integral and its relation to $M H V$ amplitudes in $\mathcal{N}=4$ SYM, JHEP 06 (2011) 100 [arXiv: 1104.2787] [INSPIRE].

[56] V. Del Duca, L.J. Dixon, J.M. Drummond, C. Duhr, J.M. Henn and V. Smirnov, The one-loop six-dimensional hexagon integral with three massive corners, Phys. Rev. D 84 (2011) 045017 [arXiv: 1105. 2011] [INSPIRE].

[57] L.J. Dixon, J.M. Drummond and J.M. Henn, to appear. 
[58] E. Remiddi and J. Vermaseren, Harmonic polylogarithms, Int. J. Mod. Phys. A 15 (2000) 725 [hep-ph/9905237] [InSPIRE].

[59] T. Gehrmann and E. Remiddi, Two loop master integrals for $\gamma^{*} \rightarrow 3$ jets: The planar topologies, Nucl. Phys. B 601 (2001) 248 [hep-ph/0008287] [INSPIRE].

[60] T. Gehrmann and E. Remiddi, Two loop master integrals for $\gamma^{*} \rightarrow 3$ jets: The nonplanar topologies, Nucl. Phys. B 601 (2001) 287 [hep-ph/0101124] [INSPIRE].

[61] T. Gehrmann and E. Remiddi, Numerical evaluation of harmonic polylogarithms, Comput. Phys. Commun. 141 (2001) 296 [hep-ph/0107173] [INSPIRE].

[62] D. Maître, HPL, a Mathematica implementation of the harmonic polylogarithms, Comput. Phys. Commun. 174 (2006) 222 [hep-ph/0507152] [INSPIRE].

[63] V. Del Duca, C. Duhr and V.A. Smirnov, The massless hexagon integral in $D=6$ dimensions, Phys. Lett. B 703 (2011) 363 [arXiv:1104.2781] [InSPIRE].

[64] B. Basso, Exciting the GKP string at any coupling, arXiv: 1010.5237 [INSPIRE]. 